\title{
Didaktische Analysen zum Bruchzahlbegriff
}

In diesem Kapitel wird die Entwicklung des Bruchzahlbegriffs als mathematischer Untersuchungsgegenstand der empirischen Studie beschrieben. Diese Beschreibung erfolgt auf der Grundlage des Grundvorstellungskonzepts (vom Hofe, 1995), das zunächst eingehend erörtert und vor dem Hintergrund der zuvor dargestellten theoretischen Transferperspektiven diskutiert wird. Im Anschluss werden Grundvorstellungen und Zahlaspekte von Bruchzahlen auf normativer Ebene beschrieben und unter Berücksichtigung zentraler empirischer Befunde Transferprozesse in der Entwicklung des Bruchzahlbegriffs abgeleitet, die als inhaltliche Strukturierung für die Unterrichtseinheit als Rahmen der empirischen Studie dienen.

Die didaktischen Analysen in diesem Kapitel verfolgen das Ziel, die Entwicklung des Bruchzahlbegriffs als Beispiel für Transfer beim Mathematiklernen zu beschreiben. Vor dem Hintergrund des im vorhergehenden Kapitel entwickelten prozessualen Verständnis von Transfer eröffnet das Grundvorstellungskonzept einen Raum für normative Analysen zur Beschreibung der didaktisch intendierten Lernprozesse in der Entwicklung des Bruchzahlbegriffs und für deskriptive Analysen zur Rekonstruktion der individuellen Lernprozesse aus den Bearbeitungen der Lernenden.

\subsection{Mathematiklernen als Ausbilden von Grundvorstellungen}

„Gelingt es einem Schüler nicht, zu wichtigen Begriffen adäquate Grundvorstellungen aufzubauen, so bleibt ihm kaum etwas anderes übrig, als sich an oberflächlichen Merkmalen, Schreibweisen oder Regeln zu orientieren. An die Stelle von Sinnkonstituierung und verständnisvoller Anwendung tritt dann ein schematisches Manipulieren; statt mit Begriffen, deren Kern er aus der Perspektive seiner Handlungs- und Vorstellungswelt mit Sinn erfüllen kann, hantiert er mit unverstandenen „Begriffshülsen“.“ (vom Hofe, 1992, S. 361) 
Damit die Anwendung mathematischer Begriffe und Operationen nicht zu einem „,unverstandenen“ Hantieren mit „Begriffshülsen“ wird, müssen Lernende mathematische Inhalte mit Sinn füllen, ihnen eine Bedeutung geben oder kurz: Eine Vorstellung zu ihnen aufbauen. Was vom Hofe (1992) in diesem Eingangszitat zum Ausdruck bringt, ist ein Gedanke, der in der deutschen Rechenmethodik der Volksschule als Vorläufer der heutigen Mathematikdidaktik eine lange Tradition hat ${ }^{1}$ : Verstehen von Mathematik wurzelt in anschaulichen Vorstellungen.

Grundvorstellungen als normative Kategorien: In seiner Konzeption von Grundvorstellungen als ,stoffdidaktische Kategorie“ (vom Hofe \& Blum, 2016) vereint vom Hofe (1995) das Anschauungs- und Vorstellungskonzept verschiedener didaktischer und psychologischer Theorien vor dem Hintergrund der Beschreibung von „Beziehungen zwischen mathematischen Inhalten und dem Phänomen der individuellen Begriffsbildung“" (vom Hofe, 1996, S. 6), die Begriffe durch Alltagsphänomene oder Handlungszusammenhänge mit Sinn erfüllen, im Gedächtnis repräsentieren und die Anwendung eines Begriffs ermöglichen (vom Hofe, 1996, S.6). Es sind besonders diese drei Aspekte, die er in seiner "Grundvorstellungsidee“ (vom Hofe, 1995, S. 97 f.) hervorhebt:

- „Sinnkonstituierung eines Begriffs durch Anknüpfen an bekannte Sach- oder Handlungszusammenhänge bzw. Handlungsvorstellungen,

- Aufbau entsprechender (visueller) Repräsentationen bzw. „Verinnerlichungen“, die operatives Handeln auf der Vorstellungsebene ermöglichen,

- Fähigkeit zur Anwendung eines Begriffs auf die Wirklichkeit durch Erkennen der entsprechenden Struktur in Sachzusammenhängen oder durch Modellieren des Sachproblems mit Hilfe der mathematischen Struktur.“

Vom Hofe beschreibt Grundvorstellungen als „Elemente der Vermittlung bzw. Objekte des Übergangs zwischen der Welt der Mathematik und der individuellen Begriffswelt des Lernenden“ (vom Hofe, 1995, S. 98). Sie vermitteln zwischen den Ebenen der Begriffe und der Gegenstände, wobei sie keinen ,statischabbildhafte[n]", sondern einen „,dynamischen Charakter" haben, denn: „Grundvorstellungen wachsen, entwickeln sich, ergänzen sich gegenseitig“" (vom Hofe, 1995, 98, Hervorhebung im Original). Ein mathematischer Begriff lässt sich in der Regel nicht mit einer isolierten Grundvorstellung erfassen, sondern nur als gegenseitige Vernetzung mehrerer Grundvorstellungen (vgl. Oehl, 1970; vom Hofe, 2003, S. 6). So kann zum Beispiel die Addition der natürlichen (ganzen) Zahlen nach Kirsch

\footnotetext{
${ }^{1}$ Eine ausführliche historische Einordnung und Darstellung der begrifflichen Genese von „Grundvorstellungen“ findet sich bei vom Hofe (1995).
} 
(1987, S. 64) wie folgt gedeutet werden (vgl. auch vom Hofe, 1995, S. 117; vom Hofe, 2003, S. 6):

- Zusammenfassen zweier Zustände zu einem neuen Zustand (Z-Z-Z), z. B.: Dirk hat 7 Spielfiguren, Mike hat 5 Spielfiguren. Wie viele haben beide zusammen? (Vereinigungs-Vorstellung)

- Änderung eines Zustands in einen neuen Zustand (Z- ̈̈-Z), z. B.: Dirk hat 7 Spielfiguren, er bekommt 5 dazu. Wie viele hat er insgesamt? (HinzufügeVorstellung)

- Hintereinanderausführen zweier Änderungen mit dem Ergebnis einer Gesamtänderung ( $\ddot{A}-\ddot{A}-\ddot{A})$, z.B.: Dirk bekommt zu seinen Spielfiguren zunächst 7, dann noch 5 weitere hinzu. Wie viele hat er insgesamt hinzubekommen? (Veränderungs-Vorstellung)

Als weiteres Beispiel beschreibt Vollrath $(1989 ; 2014)$ drei Grundvorstellungen zum Begriff einer Funktion (vgl. auch Greefrath et al. 2016):

- Eine Funktion ordnet jedem Wert einer Größe genau einen Wert einer zweiten Größe zu (Zuordnungs-Vorstellung).

- Mit Funktionen wird die Wirkung einer Änderung einer Größe auf eine zweite Größe beschrieben (Kovariations-Vorstellung).

- Eine Funktion ist ein Objekt, das einen Zusammenhang als Ganzes beschreibt (Objekt-Vorstellung).

Wie in den aufgeführten Beispielen dargestellt, sind Grundvorstellungen aus stoffdidaktischer ${ }^{2}$ Sicht normative didaktische Kategorien, ,die den Kern eines mathematischen Inhalts gemäß einer didaktischen Absicht beschreiben“" (vom Hofe, 1992, S.350). Dahinter steht die folgende Grundannahme:

\begin{abstract}
„Die individuelle Begriffsbildung kann durch didaktische Maßnahmen so unterstützt werden, daß das individuelle Begriffsverständnis den Kern des entsprechenden mathematischen Inhalts - in einem den jeweiligen Bedingungen entsprechenden Maße beinhaltet und in diesem Sinne intersubjektiv ist.“ (vom Hofe, 1992, S. 348)
\end{abstract}

Somit sind Grundvorstellungen auf normativer Ebene Leitlinien bzw.

\footnotetext{
${ }^{2}$ Zum Begriff der „Stoffdidaktik“ sei an dieser Stelle auf die Arbeiten von Griesel (1968), Kirsch (1969) und Blum (1979) verwiesen.
} 
„eine didaktische Kategorie des Lehrers, die im Hinblick auf ein didaktisches Ziel aus inhaltlichen Überlegungen hergeleitet wurde und Deutungsmöglichkeiten eines Sachzusammenhangs bzw. dessen mathematischen Kerns beschreibt.“ (vom Hofe, 1995, S. 123, Hervorhebung im Original)

Das Ziel ist es, auf Grundlage von inhaltlichen Analysen eines mathematischen Inhalts die Lernenden im Aufbau tragfähiger mentaler Repräsentationen des mathematischen Kerns zu unterstützen, die diesem eine Bedeutung geben, das Operieren auf mentaler Ebene und die Anwendung in Sachzusammenhängen ermöglichen. Diese bilden die Grundlage für die inhaltliche und methodische Planung von Lernprozessen, die es den Lernenden ermöglichen, ihre individuellen Erfahrungsbereiche zu aktivieren, um den Kern eines mathematischen Inhalts auf Grundlage der eigenen Erfahrung zu erfassen, langfristig entsprechende Grundvorstellungen aufzubauen und sie ,in das System [ihrer] Erklärungs- und Handlungsmöglichkeiten zu integrieren“ (vom Hofe, 1995, S. 125).

Grundvorstellungen als deskriptive Kategorien: Der normativen Perspektive von Grundvorstellungen stellt vom Hofe (1995) eine deskriptive Ebene der feststellbaren individuellen Vorstellungen der Lernenden gegenüber:

„Die Schülervorstellungen geben dabei Aufschluß über die individuellen Erklärungsmodelle des Schülers, die in das System seiner Erfahrungsbereiche eingebunden und entsprechend aktivierbar sind.“ (vom Hofe, 1995, S. 123, Hervorhebungen im Original)

Auf deskriptiver Ebene kann durch die Beobachtungen von Lernenden beim Arbeiten oder durch interpretierende Analysen von mündlichen oder schriftlichen Äußerungen der Lernenden eine Beschreibung ihrer individuellen Deutungen, Vorstellungen und Erklärungsmodelle vorgenommen werden und somit der Frage nachgegangen werden, was sich Lernende tatsächlich unter einem mathematischen Begriff vorstellen.

In seiner Beschreibung des deskriptiven Aspekts von Grundvorstellungen bezieht sich vom Hofe (1995) insbesondere auf Bauersfelds (1983) Modell der Subjektiven Erfahrungsbereiche (siehe Abschnitt 1.4.1) und Fischbeins (1983; 1989; 1990) Konzeption intuitiven Wissens. Ähnlich wie Bauersfeld die Subjektivität, Individualität und Bereichsspezifität von Erfahrungsbereichen beschreibt, beschreibt Fischbein eine Divergenz zwischen formalen und intuitiven Charakteristika mentaler Repräsentationen: 
„It is certainly true that, in mathematics, symbols have forcibly fixed meanings. But, when considering actual reasoning processes, one has to take into account that the subject tends to confer on the symbols his or her own interpretations. These interpretations may depend on the subject's experience, on the repertoire of accessible analogies and paradigms, and it may vary with the context.“ (Fischbein, 1990, S. 44)

Obgleich mathematische Inhalte auf formaler Ebene stets eine eng umgrenzte Bedeutung haben, bedeutet dies nicht, dass die mentalen Repräsentationen der Lernenden eben jene mathematischen Definitionen abbilden. Vielmehr sind diese geprägt von individuellen und intuitiven Annahmen ${ }^{3}$ der Lernenden, die als ,tacit models“ (vgl. Fischbein, Tirosch, Stavy \& Oster, 1990) die mentale Aktivität der Lernenden unbewusst beeinflussen und zu systematischen Fehlern führen können:

„Such errors are not slips, are not the result of a temporary lack of attention, or the result of random behavior caused by ignorance. They are generated by rules invented by the student which are different from those prescribed by mathematicians." (Fischbein, 1990, S. 44)

Lernende erfinden diese intuitiven Regeln nicht willkürlich. Stattdessen folgen sie der Logik der mentalen Zwangsläufigkeit ihrer intuitiven Annahmen und vermeintlichen Bestätigungen in praktischen Situationen: ,[...] these various, apparently unconnected rules are, in fact, generated by intuitive, primitive models inspired in turn by corresponding practical situations“" (Fischbein, 1990, S.45).

Aus diesem Grund bedarf es für eine Behebung bzw. eine Korrektur von Fehlern und ihren ursächlichen fehlerhaften mentalen Repräsentationen der Identifikation der intuitiven mentalen Modelle der Lernenden, um die Diskrepanzen zwischen formalen und intuitiven Modellen herauszustellen. Dies ist insbesondere von Bedeutung, da die intuitiven Regeln und Modelle der Lernenden ihre Handlungen leiten und aufgrund ihrer subjektiven Kohärenz sehr stabil sind:

„But a model already established is a powerful device. It may be very robust because it is coherent, it is self-consistent, and it is, usually, the first to be produced and used (the primacy effect).“ (Fischbein, 1990, S. 46)

Fischbeins Modell der unbewusst wirksamen ,tacit models“ zeigt große Ähnlichkeiten zu Bauersfelds (1983) Konzeption von subjektiven Erfahrungsbereichen. Beide

\footnotetext{
${ }^{3}$ Fischbein $(1983 ; 1989 ; 1990)$ beschreibt intuitives Wissen als spezifische Form unmittelbar verfügbaren Wissens, das keiner Begründung bedarf, sondern offensichtlich, zwangsläufig und sicher ist.
} 
Begriffe bringen zum Ausdruck, dass mentale Modelle auf Grundlage von Erfahrungen und subjektiven Wahrnehmungen gebildet werden. Eine weitere, und für die Konzeption von Grundvorstellungen entscheidende, Gemeinsamkeit der Konzeptionen von Fischbein und Bauersfeld ist, dass es sich sowohl bei intuitiven Modellen als auch bei subjektiven Erfahrungsbereichen um deskriptive Kategorien handelt. Intuitive Modelle und subjektive Erfahrungsbereiche sind keine festen Entitäten, die in den Köpfen von Individuen vorliegen und in diesem Sinne intersubjektiv sind, sondern es sind Kategorien, mit denen das Handeln von Lernenden auf Grundlage von Beobachtungen und analytischen Betrachtungen beschrieben werden kann. Durch die Analyse von schriftlichen und mündlichen Äußerungen der Lernenden kann auf ihre individuellen Erklärungsmodelle geschlossen werden und somit beschrieben werden, was sie sich unter einem Begriff, einer Operation oder allgemein mathematischen Objekten und Gegenständen vorstellen. Als Hintergrund des Grundvorstellungskonzepts bilden diese beiden Konzepte eine Grundlage für deskriptive Analysen, die einer normativen Betrachtung gegenüber gestellt werden können.

Zur Trennung des normativen und deskriptiven Aspekts von Grundvorstellungen stellt vom Hofe (1995, S.126f., Hervorhebungen im Original) im Vergleich mit subjektiven Erfahrungsbereichen heraus:

- „Individuelle Schülervorstellungen können (aus psychologischer Sicht) als Perspektiven des SEB-Systems oder vielleicht besser: als Perspektive eines koordinierenden SEB aufgefaßt werden. Grundvorstellungen als didaktische Kategorien können als inhaltliche Leit- bzw. Orientierungslinien für das „Aushandeln“ der individuellen Deutungsmöglichkeiten dienen.

- Die „Bereichsspezifität“ kann (innerhalb der stoffdidaktischen Analyse) entscheidende Hinweise auf die dem Schüler zur Verfügung stehenden Erklärungsmodelle bzw. auf die an der Lösungsstrategie beteiligten individuellen Vorstellungen geben [...].“

Durch die Gegenüberstellung der Ergebnisse von deskriptiven und normativen Analysen ist es möglich Divergenzen zwischen,,sachadäquaten Grundvorstellungen, die der Lehrer anzielt“" (vom Hofe, 1995, S. 112) und ,individuellen Vorstellungen bzw. Fehlvorstellungen“" (vom Hofe, 1995, S. 112) der Lernenden zu identifizieren. Diese dienen als Ausgangspunkt für Überlegungen zur „konstruktiven Behebung“ (vom Hofe, 1995, S. 112) möglicher Missverständnisse. 
Ausbilden von Grundvorstellungen als didaktisches Modell: In der Verbindung der normativen und deskriptiven Aspekte von Grundvorstellungen ${ }^{4}$ beschreibt vom Hofe (vom Hofe, 1995, S. 123 ff.; vom Hofe \& Blum, 2016, S. 231 ff.) das Ausbilden von Grundvorstellungen als didaktisches Modell (siehe Abb. 2.1).

In diesem didaktischen Modell stellt vom Hofe (1995, S. 124) die didaktischen Entscheidungen der Lehrenden den kognitiven Aktivitäten der Lernenden gegenüber. Der Ausgangspunkt für die didaktischen Entscheidungen ist die inhaltliche Bestimmung von adäquaten normativen Grundvorstellungen. Diese inhaltliche Bestimmung gründet sich einerseits auf Reflexionen des mathematischen Kerns, jedoch nicht allein in Form von ,eindeutigen Ableitungen“ (vom Hofe, 1995, S. 123), sondern anderseits stets unter Einbezug ,der Anwendungsdimensionen des mathematischen Inhalts und [des] Erfahrungshorizont[s]“ (vom Hofe, 1995, S. 123) der Lernenden. In der didaktischen Umsetzung werden entsprechende Sachzusammenhänge identifiziert bzw. konstruiert, die den strukturellen Kern des mathematischen Inhalts ,,in einer dem Schüler gemäßen Art repräsentieren“ (vom Hofe, 1995, S. 124).

„Die inhaltlichen Elemente und die methodische Struktur des Sachzusammenhangs bilden Ausgangspunkte für entsprechende Lern- bzw. Interaktionsprozesse und sollten dazu geeignet sein, beim Schüler Erfahrungsbereiche zu aktivieren, die es diesem ermöglichen, den Kern des Sachzusammenhangs aus der Perspektive seiner Vorstellungs- und Handlungsmöglichkeiten zu erfassen.“ (vom Hofe, 1995, S. 125)

Ziel dieses Vorgehens ist es, dass die Lernenden langfristig Vorstellungen aufbauen und entwickeln, die den normativen Kategorien, d. h. den Grundvorstellungen, möglichst entsprechen und diese in ,,das System ihrer Erklärungs- und Handlungsmöglichkeiten integrieren“ und somit am Kern des mathematischen Inhalts ,teilhaben, d.h. diesen ,verstehen“ (vom Hofe, 1995, S. 125).

In diesem Zusammenhang haben Grundvorstellungen als didaktische Kategorie eine vermittelnde Funktion, indem sie ,Beziehungen zwischen Mathematik, Individuum und Realität" (vom Hofe, 1992, S. 347) beschreiben. Das Modell verdeutlicht, dass Grundvorstellungen nicht unmittelbar von mathematischen Inhalten abgeleitet werden, sondern von Lehrenden als Orientierungsrahmen anhand inhaltlicher Refle-

\footnotetext{
${ }^{4}$ An dieser Stelle sei angemerkt, dass mit dem Begriff „Grundvorstellungen“ eine normative Kategorie als Ergebnis didaktisch orientierter Sachanalysen bezeichnet wird. Da die Vorstellungen der Lernenden im Gegensatz zu Grundvorstellungen das Ergebnis ihrer individuellen Lernprozesse sind, werden diese als (individuelle) Vorstellungen oder Individualvorstellungen und nicht als Grundvorstellungen bezeichnet. Eine ähnliche Differenzierung findet sich u. a. auch bei Greefrath et al. (2016, S. 18f.)
} 


\section{Ausbilden von Grundvorstellungen}

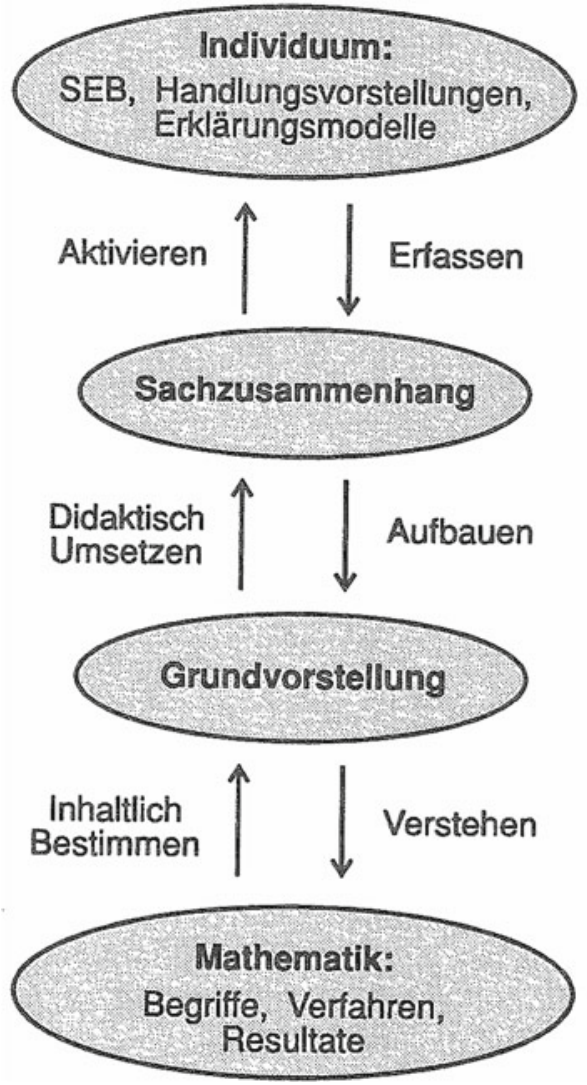

Abbildung 2.1 Modellhafte Skizze zum Ausbilden von Grundvorstellungen (vom Hofe, 1995, S. 124) als Gegenüberstellung didaktischer Entscheidungen des Lehrenden (links) und kognitiver Aktivitäten der Lernenden (rechts)

xionen bzw. stoffdidaktischer Analysen und vor dem Hintergrund der Erfahrungswelt der Lernenden mit dem Blick auf ein didaktisches Ziel formuliert werden. Das Ziel ist es, mathematische Inhalte für Lernende erfassbar zu machen. Da mathematische Inhalte nur selten in ihrer formalen und abstrakten Form Anknüpfungspunkte in der individuellen Erfahrungswelt der Lernenden bieten, ermöglichen Grundvorstellungen einen anschaulichen Zugang zu diesen Inhalten. Über die didaktische Umset- 
zung in Sachzusammenhängen soll es Lernenden möglich sein, diese auf Grundlage ihrer individuellen Erfahrungswelt zu erfassen und auf anschaulicher Ebene mental zu repräsentieren. Über die Koordination bzw. Verknüpfung verschiedener mentaler Repräsentationen und Modelle werden Vorstellungen aufgebaut, die einen anschaulichen Zugang zu den repräsentierten mathematischen Inhalten ermöglichen.

Das Ausbilden von Grundvorstellungen ist eine dynamischer Prozess. Sowohl im normativen wie im deskriptiven Sinne sind Grundvorstellungen keine Ansammlungen von statischen, ,stabilen und ein für allemal gültigen gedanklichen Werkzeugen“ (vom Hofe, 2003, S. 6). Vielmehr handelt es sich beim Ausbilden von Grundvorstellungen um

„die Ausbildung eines Netzwerks, das sich durch Erweiterung von alten und Zugewinn von neuen Vorstellungen zu einem immer leistungsfähigeren System mentaler mathematischer Modelle entwickelt.“ (vom Hofe, 2003, S. 6, Hervorhebungen im Original)

Primäre und Sekundäre Grundvorstellungen: Ein bedeutendes didaktisches Ziel des Ausbildens von Grundvorstellungen ist die Möglichkeit ein abstraktes mathematisches Konzept auf einer anschaulichen Ebene zu repräsentieren und auf diesem Wege für Lernende zugänglich zu machen, damit sie ein Verständnis für das repräsentierte mathematische Konzept entwickeln können. Mit zunehmendem Grad der Abstraktion von mathematischen Konzepten sowie der zunehmenden Komplexität mathematischer Zusammenhänge bedarf es jedoch Änderungen auf Ebene der Repräsentation dieser Konzepte. Während zum Beispiel grundlegende arithmetische Operationen mit natürlichen Zahlen, wie das Addieren, Subtrahieren, Multiplizieren oder Dividieren, als Handlungen mit Realisanten, d. h. mit konkret-gegenständlichen Objekten, realisiert werden können, ist dies etwa bei der Bestimmung der Steigung eines Funktionsgraphen in einem bestimmten Punkt nicht möglich.

Bereits vor Eintritt in den Mathematikunterricht entwickeln Kinder Vorstellungen von natürlichen Zahlen und elementaren Operationsprinzipien durch unmittelbare Handlungserfahrungen mit realen Gegenständen. Diese Handlungserfahrungen werden in der Grundschule aufgegriffen und systematisiert, zunächst mit Repräsentanten für Mengen konkreter Gegenstände, wie z. B. einem Rechenrahmen oder Mehrsystemblöcken (vgl. Wartha \& Schulz, 2012), und später mit abstrakten Repräsentationen, wie z. B. einer Zahlengeraden, anhand derer schließlich abstrakte Operationen, wie das Rechnen mit negativen Zahlen (vgl. Hattermann \& vom Hofe, 2014; vom Hofe \& Fast 2015), veranschaulicht werden können. 
Vor diesem Hintergrund wird zwischen Primären und Sekundären Grundvorstellungen unterschieden (vgl. vom Hofe \& Blum, 2016, S. 233 f.; vom Hofe, 2003, S. 6):

Primäre Grundvorstellungen basieren auf gegenständlichen Handlungserfahrungen mit realen Gegenständen und haben den Charakter von konkreten Handlungsvorstellungen. Mathematische Konzepte werden somit quasi isomorph durch Handlungen mit realen Gegenständen repräsentiert. Aus diesem Grund haben primäre Grundvorstellungen einen gegenständlichen Charakter.

Sekundäre Grundvorstellungen basieren auf mathematischen Operationen mit symbolischen Objekten. Mathematische Konzepte werden nicht anhand von realen Handlungen, sondern auf Grundlage (mentaler) Operationen mit mathematischen Objekten, wie z. B. einer Zahlengeraden, Termen oder Funktionsgraphen, repräsentiert. Sekundäre Grundvorstellungen haben daher einen symbolischen Charakter.

Die Unterscheidung zwischen primären und sekundären Grundvorstellungen steht in einer engen Beziehung zu Fischbeins (1987, S. 57 ff.) Unterscheidung zwischen primären und sekundären Intuitionen: Primäre Intuitionen entwickeln sich spontan und gehen auf persönliche Erfahrungen und das Vorwissen der Lernenden zurück. Sekundäre Intuitionen werden hingegen nicht durch natürliche Erfahrungen, sondern durch Unterricht erworben. Fischbein betont, dass Wissen, das zuerst erworben wurde, die primären Intuitionen formt. Unter dem Begriff des ,,primacy effects“ beschreibt er das Phänomen, dass das, was zuerst gelernt wurde selten vergessen wird und somit dazu führt, dass primäre Intuitionen sehr beständig sind. Aus diesem Grund existieren primäre Intuitionen zumeist neben sekundären Intuitionen und sind deshalb nicht nur ein bedeutender Einfluss für die Entwicklung von Missverständnissen, sondern auch von fehlerhaften Konzeptualisierungen (vgl. Tirosh \& Tsamir, 2014, S. 326).

Ein wesentlicher Unterschied in der Verwendung von Grundvorstellungen und Intuitionen ist, dass Grundvorstellungen vor allem als normative Kategorien der Planung von Lernprozessen verwendet werden, während Intuitionen ausschließlich als deskriptive Kategorien zur Beschreibung von individuellen Erklärungsmodellen verwendet werden. Dennoch ist das beschriebene Ziel in beiden Fällen sehr ähnlich: Primäre Grundvorstellungen bzw. primäre Intuitionen sollen im Unterricht durch sekundäre Grundvorstellungen bzw. sekundäre Intuitionen relativiert werden und somit einerseits Vorstellungen mathematischer Inhalte von der konkretgegenständlichen Handlungsebene auf die formale und symbolische Ebene des Operierens mit mathematischen Objekten überführt werden, ohne dabei die anschauliche 
Vorstellungsgrundlage aufzugeben. Anderseits sollen mögliche fehlerhafte Annahmen, die auf primären Intuitionen beruhen und im fortschreitenden Lernverlauf zu Missverständnissen und Fehlschlüssen führen können oder im fortschreitenden Lernverlauf keine Anknüpfungspunkte bieten, durch formal korrekte und tragfähige sekundäre Intuitionen und Vorstellungen ersetzt werden.

\section{Transfer aus der Perspektive von Grundvorstellungen}

Grundvorstellungen sind in erster Linie als normative didaktische Kategorien zu verstehen, die prototypisch für die Vorstellungen stehen, die bei Lernenden aufgebaut werden sollen. In seinen Ausführungen zur Nutzung von Grundvorstellungen auf einer deskriptiven Ebene hinsichtlich einer Analyse der individuellen Vorstellungen und Erklärungsmodelle der Schüler stellt vom Hofe (1995, S. 107 ff.) die Nähe zum Modell der Subjektiven Erfahrungsbereiche von Bauersfeld (1983; 1985) heraus. Auf psychologischer Ebene wird dabei die Entwicklung individueller Vorstellungen von mathematischen Inhalten im Rahmen der Entwicklung Subjektiver Erfahrungsbereiche erläutert.

Vor diesem Hintergrund beschreibt das Ausbilden von Grundvorstellungen Mathematiklernen nicht als einen kumulativen Prozess, in dem neue Vorstellungen zu bestehenden Vorstellungen hinzugefügt werden, sondern als einen dynamischen Prozess, in dem sukzessive neue Vorstellungen aufgebaut werden, Vorstellungen miteinander verknüpft, bestehende Vorstellungen erweitert und weiterentwickelt und in ein Netzwerk mentaler Repräsentationen mathematischer Inhalte integriert werden.

Die engen Kongruenzen zwischen dem Modell der Subjektiven Erfahrungsbereiche und dem Ausbilden von Grundvorstellungen erlauben auf psychologischer Ebene eine Integration hinsichtlich der Formulierung eines Erklärungsmodells für Transfer: Für einen Transfer auf neue Anforderungssituationen ist es notwendig, dass Lernende in diesen Situationen bestehende Erfahrungsbereiche bzw. Vorstellungen aktivieren, die in einem neuen koordinierenden Erfahrungsbereich miteinander verknüpft werden. Dieser neu entstandene koordinierende Erfahrungsbereich ermöglicht es den Lernenden eine neue Situation zu erfassen und mit Bedeutung zu füllen und schließlich entsprechende Handlungen durchzuführen bzw. Wissen zu übertragen und anzuwenden. Diese Prozesse sind in vom Hofes didaktischem Modell (vgl. Abb. 2.1) in den Schritten der Erfassung von Sachzusammenhängen und dem Aufbau von Grundvorstellungen einzuordnen, die letztendlich das Verstehen von mathematischen Begriffen, Verfahren und Zusammenhängen unterstützen sollen. Gleichzeitig greifen Lernende für die Erfassung (neuer) Sachzusammenhänge auf ihr individuelles Netzwerk an bestehenden Erfahrungsbereichen und Vorstellungen zurück, sodass in diesem Zusammenhang eine gegenseitige Bedin- 
gung zwischen der Ausbildung von Grundvorstellungen und Transfer angenommen werden kann.

In einer Diskussion zur Bedeutsamkeit von Grundvorstellungen für die allgemeine mathematische Kompetenzentwicklung am Beispiel der Modellierungskompetenz resümieren vom Hofe und Blum (2016):

„These relationships demonstrate the important role of GVs [Grundvorstellungen] for the development of mathematical competencies. Ideally, this development will be accompanied by the formation of both primary GVs and, with progressive learning, increasingly also secondary GVs, into a growing and networked system. In particular, the ability to apply mathematical skills is based, according to this view, on the quality of development and the degree of cross-linking of GVs, as well as on the ability to activate and coordinate GVs.“" (vom Hofe \& Blum, 2016, S. 235 f., Hervorhebung des Autors)

In diesem Beitrag beschreiben vom Hofe und Blum (2016) die Notwendigkeit von Grundvorstellungen im Rahmen der Anwendung in Sachkontexten - bzw. Transfer in einem klassischen Begriffsverständnis - und mathematischen Modellierungssituationen insbesondere in folgenden Bereichen (vgl. vom Hofe \& Blum, 2016, S. 234 ff.):

- Dem Übersetzen zwischen Mathematik und Realität als Interpretation von realen Situationen mit mathematischen Konzepten und umgekehrt,

- dem Übersetzen zwischen verschiedenen Darstellungen (z. B. geometrischen und algebraischen Darstellungen) und

- dem Begründen bzw. prä-formalen Beweisen mathematischer Zusammenhänge.

In diesen exemplarischen Anwendungsbereichen von Grundvorstellungen lassen sich die Prozesse des Verknüpfens sowie des Ausbildens und Erweiterns von Vorstellungen bzw. Erfahrungsbereichen der Lernenden nachzeichnen.

In verschiedenen empirischen Untersuchungen (vgl. z. B. Pekrun et al., 2006; Wartha, 2007; Hafner, 2012) konnte nachgewiesen werden, dass wesentliche Schwierigkeiten in der Entwicklung mathematischer Kompetenzen und Fähigkeiten auf Defizite in der Entwicklung von adäquaten Grundvorstellungen zurückzuführen sind. Im Speziellen zeigt die Studie zur Leistungsentwicklung in der Bruchrechnung von Wartha (2007) auf, dass der Aufbau von Grundvorstellungen insbesondere im fortschreitenden Verlauf der Begriffs- und Kompetenzentwicklung nicht ausreicht. In seinen Fallstudien zeigte Wartha auf, dass die Zahlbereichserweiterung von den natürlichen zu den positiven rationalen Zahlen gravierende Veränderungen von aus der Grundschule vertrauten Grundvorstellungen erfordert. Sowohl hinsichtlich der 
Entwicklung des Bruchzahlbegriffs sowie zu den Grundrechenarten müssen neue Aspekte in das bestehende Vorstellungsgefüge integriert und bisherige, im Bereich der natürlichen Zahlen gültige Aspekte verändert, erweitert und zum Teil verworfen werden (vgl. Wartha \& vom Hofe, 2005, S. 10). Diese sogenannten Grundvorstellungsumbrüche und die Entwicklung von Fehlvorstellungen können im Wesentlichen durch ausbleibenden oder negativen Transfer, z. B. der Übertragung der Ordnungsstruktur der natürlichen Zahlen auf die Bruchzahlen, interpretiert werden. Zudem ist die ausbleibende Anpassung von Vorstellungen durch die Stabilität von Vorwissensstrukturen erklärbar (siehe oben).

Hinsichtlich der Nutzung des Grundvorstellungskonzepts zur „konstruktiven Analyse" (vom Hofe, 1995 , S. 113) von Lern- und Bearbeitungsprozessen erläutert vom Hofe (vom Hofe, 1995, $116 \mathrm{f}$., Hervorhebungen im Original):

„Eine umfassende Erklärung der Schülerstrategie und der Mißverständnisse, die sich angesichts des vom Lehrer erwarteten bzw. vom Schüler eingeschlagenen Lösungsversuchs ergeben, bringt eine Analyse der normativ verwendeten Grundvorstellungen und der deskriptiv feststellbaren Individualvorstellungen, etwa unter den Leitfragen:

- Welche Grundvorstellungen sind zur Lösung des Problems aus Sicht des Lehrenden adäquat? (Normativer Aspekt)

- Welche individuellen Vorstellungen lassen sich im Lösungsversuch des Schüler erkennen? (Deskriptiver Aspekt)

- Worauf sind etwaige Divergenzen zurückzuführen, und wie lassen sich diese beheben? (Konstruktiver Aspekt)“

Aus einer didaktischen Perspektive erscheint dieser Ansatz auch geeignet, die Bearbeitungen von Transferaufgaben zu untersuchen. Auf normativer Ebene lassen sich vor dem Hintergrund des vorhergehenden Unterrichts sowie den vermuteten Erfahrungen der Lernenden Grundvorstellungen zum mathematischen Kern eines Transfers ableiten. Hierbei wird angenommen, dass diese Vorstellungen eine Übertragung der entsprechenden mathematischen Inhalte auf neue Anforderungssituationen ermöglichen und unterstützen. Gleichzeitig ist jedoch denkbar, dass nicht adäquate, nicht hinreichend entwickelte oder auch fehlerhafte Vorstellungen den Transfer erschweren und möglicherweise verhindern können. Deskriptive Analysen individueller Erklärungsmodelle der Lernenden geben Aufschluss über mögliche Ursachen für einen fehlerhaften oder ausbleibenden Transfer und stellen somit eine Grundlage für die Planung konstruktiver Unterstützungsmaßnahmen dar.

Die differenzierte Analyse der individuellen Erklärungsmodelle der Lernenden steht auch im Mittelpunkt der im vorhergehenden Abschnitt diskutierten Perspektive des Actor-Oriented Transfer. In der AOT Perspektive wird argumentiert, dass Ler- 
nende auf Grundlage ihrer subjektiven Wahrnehmung und individuellen Erfahrung Generalisierungen vornehmen, die ihr Handeln in Transfersituationen leiten und sich in ihren individuellen Erklärungsmodellen niederschlagen. Im Rahmen dieser Perspektive können Grundvorstellungen als Perspektive des Beobachters bzw. des Experten interpretiert werden. Sie beschreiben von der formalen mathematischen Ebene ausgehend Vorstellungen und mentale Modelle, die aus Sicht von Experten bzw. der Lehrenden zu einem mathematischen Inhalt ausgebildet werden sollten. Die in der AOT Perspektive beschriebenen individuellen Generalisierungen der Lernenden können aus Perspektive des Grundvorstellungskonzepts als Erfahrungsbereiche oder individuelle Vorstellungen interpretiert werden, die in der individuellen Auseinandersetzung mit mathematischen Inhalten entstehen.

Insgesamt kann das Grundvorstellungskonzept als ,ganzheitliche Sichtweise mathematischen Denkens und Handelns“" (vom Hofe, 1995, S. 131) bezeichnet werden. Durch die Zusammenführung von sachlogischen und psychologischen Aspekten des Mathematiklernens eröffnet es einen Rahmen zur Integration anderer Konzepte. Obgleich der Transfer mathematischen Wissens und mathematischer Fähigkeiten in der Literatur zu Grundvorstellungen lediglich im Zusammenhang mit der Anwendung von Mathematik in Modellierungssituationen diskutiert wurde, ist ein immanentes Ziel der Ausbildung von Grundvorstellungen darin zu erkennen, dass Lernende mathematische Begriffe, Handlungen und Strukturen anwenden und somit auch auf neue Sachzusammenhänge übertragen können.

Als ein didaktisches Modell ist das Grundvorstellungskonzept primär zum Einsatz in der Unterrichtspraxis ausgerichtet: Stoffdidaktische Analysen mathematischer Inhalte ermöglichen die Konstruktion von Zugängen für Lernende, die einerseits vom mathematischen Kern abgeleitet werden und gleichzeitig die Erfahrungswelt der Lernenden und damit ihre individuellen Voraussetzungen einbeziehen.

Als ein empirisches Modell zur deskriptiven Analyse von individuellen Vorstellungen der Lernenden liefert es Ansatzpunkte für die Untersuchung spezifischer Fragestellungen in einem unterrichtsnahen Kontext. Die Offenheit gegenüber anderen didaktischen und psychologischen Erklärungsmodellen eröffnet einen Rahmen für die empirische Untersuchung spezifischer mathematikdidaktischer Fragestellungen.

Im Fazit der Darstellung und Diskussion der verschiedenen theoretischen Perspektiven zum Transfer wurde festgestellt, dass Transfer als dynamischer Prozess der Übertragung einer Wissensstruktur auf ein neues Anwendungsgebiet beschrieben werden kann, bei dem sowohl die Art der übertragenen Wissensstruktur sowie der Ursprung und das Ziel der Übertragung eindeutig definiert werden können. Vor dem Hintergrund des Grundvorstellungsmodells kann diese Definition von Transferprozessen in Hinsicht auf das Mathematiklernen weiter präzisiert werden: 
Ein Transferprozess ist der Prozess der Anwendung oder Übertragung mathematischer Begriffe, Verfahren und Strukturen auf eine neue Anforderungssituation

- zum Transfer zwischen Sach- und Anwendungskontexten,

- zum Transfer zwischen verschiedenen Darstellungen und Repräsentationsebenen sowie

- zum Herstellen und Begründen von mathematischen Zusammenhängen.

Die Anwendung oder Übertragung mathematischer Begriffe, Handlungen (Verfahren) und Strukturen erfordert die Aktivierung von Grundvorstellungen und trägt dadurch wechselseitig zum Aufbau, zur Entwicklung und zur Verknüpfung von Grundvorstellungen bei.

Durch die Bezugnahme auf die Ausbildung von Grundvorstellungen ist es möglich auf normativer Ebene Transferprozesse auf der Basis sachanalytischer Überlegungen in Hinsicht auf ein didaktisches Ziel zu beschreiben und auf deskriptiver Ebene in den Bearbeitungen der Lernenden zu analysieren. In diesem Zusammenhang können die Leitfragen zur Analyse der normativen Verwendung von Grundvorstellungen und der deskriptiv feststellbaren Individualvorstellungen der Lernenden neu formuliert werden:

- Welche Transferprozesse sind zur Lösung einer neuen Anforderung erforderlich und welche Grundvorstellungen werden dafür benötigt? (Normativer Aspekt)

- Welche individuellen Transferprozesse lassen sich in den Bearbeitungen der Lernenden erkennen und welche individuellen Vorstellungen liegen diesen zugrunde? (Deskriptiver Aspekt)

- Worauf sind etwaige Divergenzen zurückzuführen und wie lassen sich diese beheben? (Konstruktiver Aspekt)

Im folgenden Abschnitt werden Grundvorstellungen zu Bruchzahlen und zum Operieren mit Bruchzahlen auf Grundlage sachanalytischer Überlegungen dargestellt und hinsichtlich empirischer Befunde, insbesondere zu Problembereichen in der Entwicklung des Bruchzahlbegriffs, diskutiert.

\subsection{Grundvorstellungen zum Bruchzahlbegriff}

Die Zahlbereichserweiterung von den natürlichen Zahlen zu den rationalen Zahlen ist einer der zeitintensivsten und zugleich herausforderndsten Bereiche im Mathematikunterricht der Sekundarstufe I, denn 
„wegen des erstmals höheren Abstraktionsgrades passiert es hier durchaus nicht selten, dass bei Lernenden erstmals das mathematische Verständnis weitgehend auf der Strecke bleibt und an seine Stelle das blinde Auswendiglernen unverstandener und darum leicht zu verwechselnder Regeln tritt.“ (Padberg \& Wartha, 2017, S. V).

Dies ist insbesondere problematisch, da ein verständiger Umgang mit Bruchzahlen eine wesentliche Voraussetzung für weiterführendes Lernen in nahezu allen mathematischen Inhaltsbereichen ist ${ }^{5}$ (vgl. z. B. Bailey, Hoard, Nugent \& Geary, 2012; Siegler et al., 2012; Torbeyns, Schneider, Xin \& Siegler, 2015). Bruchzahlen sind als positive rationale Zahlen definiert. Die Einführung im Mathematikunterricht erfolgt in der Regel vor der Erweiterung der ganzen Zahlen. Lediglich wenn diese vor der Zahlbereichserweiterung auf die Bruchzahlen erfolgt ist, werden in der ersten systematischen Einführung von Brüchen auch negative rationale Zahlen behandelt (vgl. Wartha, 2007, S. 41 f.; Griesel, 1970).

Mit der Einführung von Bruchzahlen werden im Allgemeinen die Ziele verfolgt, dass Schülerinnen und Schüler

- „Probleme aus Umweltsituationen, in denen die Begriffe der Bruchrechnung zur Anwendung kommen, versteh[en], sie beschreiben und lösen [können],

- Einsicht in die Rechenregeln und Gesetze der Bruchrechnung gewinn[en] und damit die Regeln und Gesetze nicht nur rezeptartig anwende[n].“ (Postel, 1981, S. 16)

Dies setzt voraus, dass die Schülerinnen und Schüler tragfähige und sachadäquate Vorstellungen von den Begriffen der Bruchrechnung aufbauen. Im Folgenden werden die zentralen Grundvorstellungen und Zahlaspekte von Bruchzahlen genauer beschrieben und erörtert ${ }^{6}$.

\footnotetext{
${ }^{5}$ Eine Übersicht über Anwendungen der Bruchrechnung in anderen mathematischen Inhaltsbereichen findet sich u. a. bei Padberg und Wartha (2017, S. 8 ff.). Auf empirischer Ebene berichten kognitionspsychologische Studien zudem eine signifikante Vorhersagekraft von Kompetenzen im Bereich der Bruchrechnung auf den späteren Lernerfolg in Mathematik

${ }^{6}$ Vor dem Hintergrund des Forschungsinteresses beschränkt sich die folgende Darstellung auf für die Studie relevante Zahlaspekte und Grundvorstellungen. Ausführungen zum Rechnen mit Bruchzahlen finden sich u. a. bei Wartha (2007), Malle (2004) und Postel (1981). Es sei zudem auf die Sachanalysen des mathematischen Inhalts von Griesel (1970) und Kirsch $(1970,1987)$ verwiesen.
} 


\subsubsection{Aspekte des Bruchzahlbegriffs}

Entgegen einer abstrakten Konstruktion der rationalen Zahlen, die wissenschaftlich über die Bildung von Äquivalenzklassen von Zahlenpaaren natürlicher oder ganzer Zahlen erfolgt, orientiert sich die Behandlung von Bruchzahlen in der Schule an Anwendungsaspekten und anschaulichen Alltagsphänomenen. Hinsichtlich der verschiedenen Anwendungsaspekte kann zwischen acht Zahlaspekten von Bruchzahlen unterschieden werden (vgl. Padberg \& Wartha, 2017, S. 17 ff.; Postel, 1981, S. 17 f.; Malle, 2004), die Teilbereiche des Bruchzahlbegriffs beschreiben und diesen fachlich charakterisieren.

Bruch als Anteil: Die Interpretation eines Bruchs als Anteil ist ein fundamentaler Bruchzahlaspekt. Demnach bedeutet $\frac{3}{4}$, dass eine Bezugsgröße restlos in vier gleichgroße Teile aufgeteilt und drei dieser Teile ausgewählt werden. In Abhängigkeit von der Bezugsgröße wird zwischen einem Bruch als Teil eines Ganzen und einem Bruch als Teil mehrerer Ganzer unterschieden. Die Bezugsgröße bzw. das Ganze kann ein Objekt (z. B. eine Pizza, ein Kuchen, etc.), eine kontinuierliche Größe (z.B. eine Strecke, eine Fläche, etc.) oder eine diskrete Größe (z. B. eine Menge von 30 Personen, ein Geldbetrag von $200 €$, etc.) sein. Anteile können an unterschiedlichen Repräsentanten ikonisch und enaktiv dargestellt werden und weisen vielfältige Alltagsbezüge auf (vgl. Padberg \& Wartha, 2017, S. 19f.; Hefendehl-Hebeker, 1996, Malle, 2004, S.4).

Einige Autoren (z. B. Malle, 2004, S.4; Schink, 2013, S. 25 ff.) unterscheiden zwischen einem relativen und einem absoluten Anteil. So wird argumentiert, dass etwa der Anteil $\frac{2}{3}$ von $900 €$ den Anteil an einer Gesamtbezugsgröße $(900 €)$ angibt, auf den sich der Anteil bezieht. Demgegenüber beschreibt ein absoluter Anteil eine Relation zwischen zwei Größen, die keine Informationen über die Bezugsgröße bzw. Gesamtanzahl geben. Diese Angabe wird häufig zur Beschreibung relativer Häufigkeiten verwendet, bei denen die Gesamtbezugsgröße (3 Teile) und die Anteilsbezugsgröße (2 Teile) in Relation gesetzt werden. In dieser Arbeit werden die Ausdrücke relativer und absoluter Anteil jedoch mit einer anderen Bedeutung verwendet. Der Begriff relativer Anteil wird für Bruchoperatoren und der Begriff absoluter Anteil für den mit einem Bruchoperator bestimmten Bruchteil verwendet. Im Beispiel $\frac{2}{3}$ von $900 €=600 €$ wird mit $\frac{2}{3}$ der relative Anteil und das Ergebnis $600 €$ als absoluter Anteil von $900 €$ bezeichnet.

Bruch als Maßzahl: Bestimmt ein Bruch einen Anteil an einer normierten Maßeinheit in einem Größenbereich (z. B. Längen, Gewichte, Volumina, Flächeninhalte, Zeiteinheiten, etc.) so wird der Bruch als Maßzahl interpretiert und zum Teil auch 
als ,konkreter Bruch“ bezeichnet. Maßzahlen treten zumeist in Sachsituationen auf und bezeichnen in Kombination mit einer Maßeinheit eine Größe (z. B. $\frac{1}{2} \mathrm{~km}, \frac{3}{4} \mathrm{~kg}$, $\left.\frac{1}{4} h\right)$. Durch ihre Einbettung in Sachkontexte ergeben sich Bezüge zum Alltag der Lernenden. Repräsentanten für Größen sind dabei häufig Strecken, Flächen, geometrische Körper, physikalische Körper oder Ereignisse (vgl. Griesel, 1970, S.6; Postel, 1981, S. 17; Wartha, 2007, S. 49; Padberg \& Wartha, 2017, S. 20).

Bruch als Operator: Bei der Interpretation von einem Bruch als Operator, repräsentiert der Bruch eine Funktion, die multiplikativ auf eine Zahl oder Größe wirkt. Ein Bruchoperator ist die Zusammenfassung zweier Teiloperatoren, dem Zählerund dem Nenneroperator (Griesel, 1981c). Der Zähleroperator (Faktor im Zähler) bewirkt eine Vervielfachung (Streckung) der Eingabe, während der Nenneroperator (Divisor im Nenner) eine Verkleinerung (Stauchung) der Eingabe bewirkt. Aus diesem Grund kann das Ergebnis als eine Verkettung der beiden Teiloperatoren kleiner oder größer als die Ausgangsgröße sein. Die Verkettung der beiden Teiloperatoren entspricht der Multiplikation mit der Bruchzahl.

In diesem Sinne werden durch die Deutung eines Bruchs als Operator auf symbolischer Ebene auf Größen anzuwendende Rechenanweisungen bzw. auf ikonischer und enaktiver Ebene (Repräsentantenebene) entsprechende Handlungsanweisungen beschrieben. Somit kann mit diesem Aspekt u. a. der „Bruchherstellungsakt“ (Postel, 1981, S. 23) bzw. der dynamische Prozess der Bruchherstellung beschrieben werden. Sollen zum Beispiel $\frac{3}{4}$ von $1 \mathrm{~cm}^{2}$ bestimmt werden, wird der Bruchoperator ,$\frac{3}{4}$ von“" als Rechen- bzw. Handlungsanweisung ,teile durch 4“ und ,,vervielfache mit 3“ interpretiert. Bei der Hintereinanderausführung der beiden Teiloperatoren hat die Reihenfolge, in der die Teiloperatoren angewendet werden, keinen Einfluss auf das Ergebnis (vgl. Postel, 1981, S.23; Griesel, 1981c; Wartha, 2007, S. 54 ff.).

Bei der Interpretation eines Bruchs als Vergleichsoperator wird unter dem Relationsaspekt ein Bezug zwischen zwei Größen hergestellt und als Bruchzahl interpretiert (vgl. Postel, 1981, S.17; Malle, 2004, S.4f.). Dabei können zwei Fälle unterschieden werden:

(1) Eine Größe wird auf eine andere Größe derselben Art bezogen, z. B. „Fleisch besteht zu $\frac{2}{3}$ aus Wasser“ (Postel, 1981, S. 17). Hierbei gibt die Bruchzahl den Anteil des Wassers im Fleisch an und stellt somit eine Relation zwischen den Gewichten des Wassers und des Fleisches her. In diesem Fall ist die Bruchzahl kleiner als Eins.

(2) Eine Größe wird auf dieselbe Größe bezogen, z. B. „Die Ernteerträge betrugen in diesem Jahr das $1 \frac{1}{2}$-fache des Vorjahres“ (Postel, 1981, S. 17). In diesem Fall kann die Bruchzahl größer als Eins sein. 
(1)

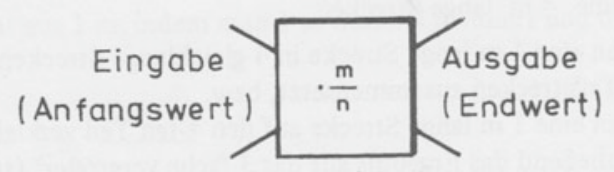

(2)

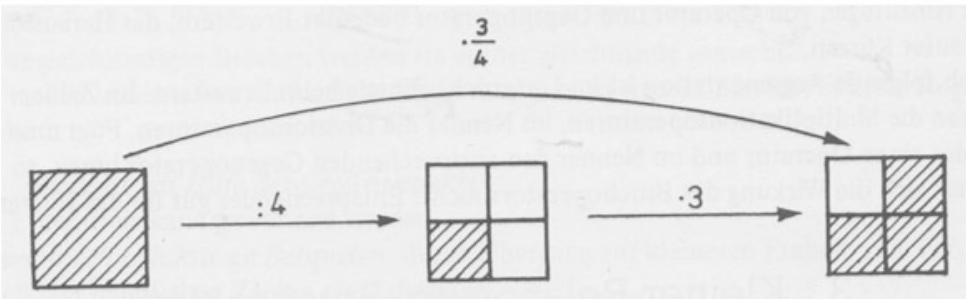

(3)

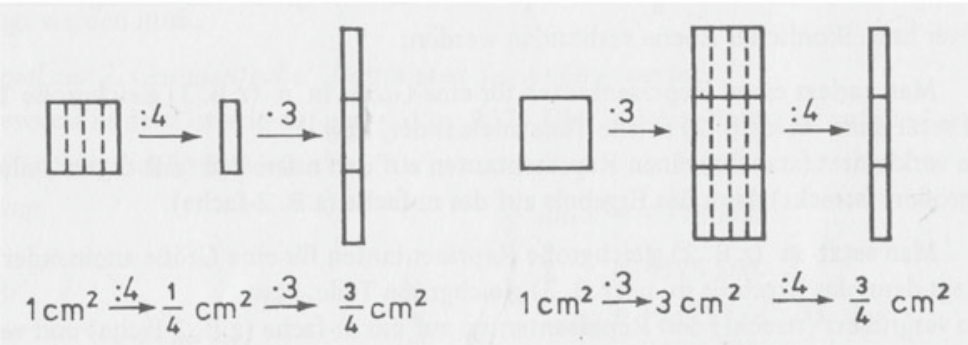

Abbildung 2.2 (1) Bruchoperator als Maschine, (2) Bruchherstellungsakt als Hintereinanderausführung zweier Teiloperatoren, (3) Vertauschen der Teiloperatoren in der Bruchherstellung ändert den Anteil nicht (Postel, 1981, S. 24, 23, 29)

Bruch als Quasikardinalzahl und Quasiordinalzahl: Beim quasikardinalen Zahlaspekt von Brüchen wird der Nenner eines Bruchs $\frac{m}{n}$ als Einheit aufgefasst, sodass der Bruch als Größe mit der Maßzahl $m$ und der Größeneinheit $\frac{1}{n}$ interpretiert wird. Schreibt man zum Beispiel den Bruch $\frac{3}{4}$ in der Form 3 Viertel, so wird 3 als Kardinalzahl analog zum exemplarischen Ausdruck 3 Bäume behandelt: Die Größeneinheiten sind jeweils „Viertel“ und „Bäume“. Der Quasikardinalzahlaspekt führt Bruchzahlen somit direkt auf natürliche Zahlen zurück. Durch die Deutung des Nenners als Maßeinheit ist der Quasikardinalzahlaspekt eng mit dem Maßzahlaspekt verbunden und ,es gehört damit zwingend zum Begriff des Halben, des Drittels oder des Viertels, dass alle Hälften (Drittel, Viertel) jeweils gleich groß sind“" (Griesel, 1981b, S.92). Die Prozesse der anschaulichen Herstellung eines Bruchs als Anteil 
und einer Quasikardinalzahl sind somit dieselben (vgl. Griesel, 1981b; Malle, 2004; Wartha, 2007, S. 49f.; Padberg \& Wartha, 2017, S. 21).

Analog kann im Spezialfall der Stammbruchherstellung der Bruch $\frac{1}{n}$ als Quasiordinalzahl interpretiert werden. So kann zum Beispiel die Aussage „Jedes fünfte Auto ist schwarz" bedeuten, dass im strikten Sinne, jedes fünfte Auto, dass vorbeifährt schwarz ist oder im statistischen Sinne, dass ein Fünftel aller vorbeifahrenden Autos schwarz sind (vgl. Freudenthal, 1983; Malle, 2004, S. 5; Wartha, 2007, S. 50 f.).

Bruch als Quotient: Ein Bruch $\frac{m}{n}$ kann als Quotient $m: n$ und somit als Resultat einer Division aufgefasst werden. Zum Beispiel kann in Verbindung mit der Interpretation eines Bruchs als Teil mehrerer Ganzer das Aufteilen von drei Pizzen auf vier Personen als Division 3 : 4 gedeutet werden und somit unmittelbar bestimmt werden, dass jede Person den Anteil $\frac{3}{4}$ einer Pizza erhält (Malle, 2004, S. 5). In engem Zusammenhang dem Quotientenaspekt steht die Interpretation eines Bruchs als Lösung einer linearen Gleichung. So kann der Bruch $\frac{2}{3}$ als Lösung der linearen Gleichung $3 \cdot x=2$ aufgefasst werden, die durch die Division $2: 3$ erhalten wird (Padberg \& Wartha, 2017, S. 21).

Bruch als Verhältnis: Die Interpretation eines Bruchs als Verhältnis stellt eine Verbindung zum Konzept der Proportionalität her. Die Angabe eines Verhältnisses bezeichnet in der Regel ein inneres Teilverhältnis. In selteneren Fällen werden äußere Teilverhältnisse beschrieben, z. B. ,,von je drei aufeinanderfolgenden Perlen ist eine schwarz, jeweils zwei von drei aufeinanderfolgenden Perlen sind weiß“ (Wartha, 2007, S. 59). Ein inneres Teilverhältnis $2: 3$ kann in inhaltlich gleichwertige Bruchangaben umgewandelt werden, sodass $\frac{2}{5}$ und $\frac{3}{5}$ die Anteile beschreiben. Das Arbeiten mit Verhältnissen beschränkt sich zumeist auf den Vergleich mehrerer Verhältnisse oder die Zusammenführung zweier Verhältnisse, z. B.: Zwei Saftschorlen mit unterschiedlichen Mischungsverhältnissen werden gemischt. Wie ist das neue Mischungsverhältnis? (vgl. Wartha, 2007, S. 59f.; Malle, 2004, S. 5).

Bruch als Skalenwert Werden Bruchzahlen zur Bezeichnung von Stellen auf einer Skala (z. B. einer Tankskala) oder von Punkten auf einer Halbgeraden (Zahlenstrahl) und somit als Koordinaten einer markierten Stelle verwendet, spricht man von einem Bruch als Skalenwert. Auf symbolischer Ebene wird mit einem Bruch als Skalenwert ein konkreter Anteil beschrieben: Der Nenner beschreibt die Einteilung der Skala und der Zähler die Anzahl der betrachteten Segmente. Dieser Bruchzahlaspekt steht in einem engen Zusammenhang mit dem Maßzahlaspekt (vgl. Postel, 1981, S. 18; Wartha, 2007, S. 51; Padberg \& Wartha, 2017, S. 21). 
Vernetzungen der Bruchzahlaspekte: Wie in den Beschreibungen der verschiedenen Zahlaspekte von Brüchen bereits angemerkt wurde, sind diese nicht in Abgrenzung voneinander zu betrachten, sondern zeichnen sich durch inhaltliche Überschneidungen und Verbindungen aus (Wartha, 2007, S. 65). Bereits eine Veränderung der Betrachtungsweise einer Bruchzahl kann zu einer Veränderung des dominierenden Zahlaspekts führen (vgl. Hefendehl-Hebeker, 1996, S. 20). Padberg und Wartha (2017, S. 19) bezeichnen die unterschiedlichen Zahlaspekte aus diesem Grund als „unterschiedliche Verwendungssituationen“ von Brüchen und lösen sie somit aus dem strengen fachlichen Kontext. Im amerikanischen Raum werden Bruchzahlaspekte vor allem als ,,subconstructs“ rationaler Zahlen bezeichnet (vgl. Kieren, 1993, S.57; Steffe \& Olive, 2010; Hackenberg \& Lee, 2016; Reinhold, 2019).

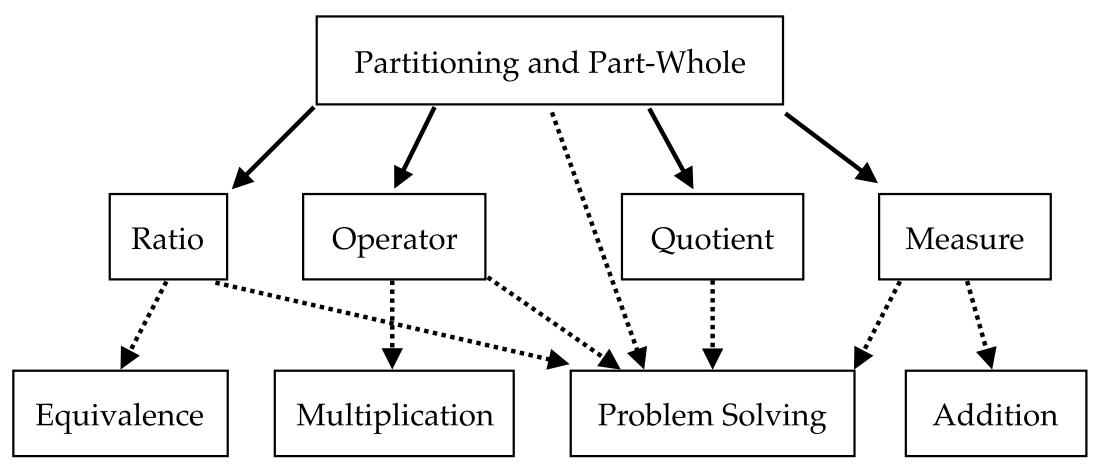

Abbildung 2.3 Beziehungen zwischen Bruchzahlaspekten nach Behr et al. (1983, S.99).

Behr et al. (1983, S. $99 \mathrm{ff}$.) beschreiben in einem Modell die Zusammenhänge unterschiedlicher Bruchzahlaspekte, in das sie auch die Operationen mit Bruchzahlen miteinbeziehen. Darin beschreiben sie den Anteilaspekt als grundlegenden Bruchzahlaspekt, aus dem die Teilaspekte Verhältnis, Operator, Quotient und Maßzahl abgeleitet werden. Nach diesem Modell ist der Verhältnisaspekt der Ausgangspunkt für Erkennen von und Arbeiten mit äquivalenten Brüchen (in Abb. 2.3 durch gestrichelte Pfeile gekennzeichnet), der Operatoraspekt motiviert und erklärt die Multiplikation und der Maßzahlaspekt ist eine natürliche Grundlage für die Addition von Brüchen. Alle Aspekte sind gleichsam für das Problemlösen mit Bruchzahlen relevant. Dieses Modell soll an dieser Stelle lediglich illustrativ als Beispiel für die beschriebenen Zusammenhänge zwischen Bruchzahlaspekten dienen. Vielmehr 
wurde in diesem Abschnitt herausgestellt, dass Bruchzahlen ,,vielfältige Erscheinungsformen“ (Hefendehl-Hebeker \& Schwank, 2015, S. 101) haben, die einerseits aus fachlichen Charakterisierungen abgeleitet werden können und in verschiedenen Anwendungszusammenhängen verschiedene Interpretationsmöglichkeiten von Bruchzahlen darstellen. In Hinsicht auf die Entwicklung eines Verständnisses von Bruchzahlen ist es von besonderer Bedeutung, verschiedene Zahlaspekte miteinander in Beziehung zu setzen, ,da sich das Verständnis eines mathematischen Begriffs nicht aus der Summe der Einzelaspekte ergibt" (Wartha, 2007, S. 49).

Im Zusammenhang mit dem Ausbilden von Grundvorstellungen als didaktische Kategorien können Bruchzahlaspekte zudem als Teilaspekte von Grundvorstellungen gesehen werden, wie es im folgenden Abschnitt beschrieben wird.

\subsubsection{Grundvorstellungen von Bruchzahlen}

Es lassen sich drei zentrale Grundvorstellungen ${ }^{7}$ zum Bruchzahlbegriff unterscheiden, die den verschiedenen Bruchzahlaspekten zugrunde liegen und zu ihrem Verständnis beitragen (Wartha, 2007, S. 48; vgl. auch Padberg \& Wartha, 2017, S. 21 ff.):

Bruch als Anteil: $\frac{m}{n}$ als $m$ von $n$ Teilen

Bruch als Operator: Eine Menge wird auf das $\frac{m}{n}$-fache der Menge abgebildet Bruch als Verhältnis: $m: n$ als Relation zwischen $m$ Teilen und $n$ Teilen

Ähnlich wie in der Beschreibung der verschiedenen Bruchzahlaspekte sind auch diese drei Grundvorstellungen nicht überschneidungsfrei voneinander zu trennen. In ihrem Kern beschreiben sie dennoch drei unterschiedliche mentale Modelle von Bruchzahlen. Im Folgenden werden die Anteil- und die Operatorvorstellung näher beschrieben. Auf eine eingehende Darstellung der Verhältnisvorstellung wird an dieser Stelle verzichtet, da diese ,,als Bindeglied zum Inhaltsbereich der Proportionalität" (Wartha, 2007, S. 59) zu sehen ist und für die Entwicklung des Bruchzahlbegriffs keine tragende Rolle einnimmt.

Grundvorstellung Bruch als Anteil: Im Alltag treten Brüche zumeist als Anteile auf und bieten daher zahlreiche Anknüpfungspunkte an die Erfahrungswelt der

\footnotetext{
${ }^{7}$ In der Literatur finden sich unterschiedliche Modelle zu Grundvorstellungen zu Bruchzahlen (z. B. Malle, 2004). Die Darstellung in dieser Arbeit bezieht sich auf die Differenzierung von Wartha (2007), die zwischen Zahlaspekten als fachliche Kategorien und Grundvorstellungen als didaktische Kategorien unterscheidet.
} 
Schülerinnen und Schüler. Unter der Anteilvorstellung lassen sich verschiedene Bruchzahlaspekte vereinen (vgl. Wartha, 2007, S. 49 ff.):

1 Bruch als Teil eines Ganzen,

2 Bruch als Teil mehrerer Ganzer,

3 Bruch als Maßzahl,
4 Bruch als Quasikardinalzahl,

5 Bruch als Quasiordinalzahl und

6 Bruch als Skalenwert.

Eine konstituierende Rolle nehmen die Zahlaspekte Bruch als Teil eines Ganzen und Bruch als Teil mehrerer Ganzer ein. Brüche als Anteile können an unterschiedlichen Objekten veranschaulicht werden: Das Ganze kann dabei ein konkreter Gegenstand, z. B. eine Pizza oder ein Kuchen, eine kontinuierliche (zusammenhängende) Größe, z. B. eine Fläche oder eine Länge, oder eine diskrete (getrennte) Größe, wie. z.B. eine Menge von Puzzleteilen, sein. In jedem Fall stellt die Grundvorstellung des Bruchs als Anteil eine Relation zwischen Teil und Ganzem her (Wartha \& Güse, 2009, S. 263; vgl. auch Schink, 2013, S. 25 ff.): $\frac{3}{4}$ Kuchen bedeutet, dass ein Kuchen in vier gleich große Teile geteilt wird, von denen drei betrachtet werden. In diesem Beispiel ist der Anteil $\frac{3}{4}$ eine Zahl, die den Zusammenhang zwischen einem Teil des Kuchens und einem ganzen Kuchen beschreibt: $\frac{3}{4}$ Kuchen ist das dreifache des vierten Teils des ganzen Kuchen (vgl. „Relation“ bei Postel, 1981, S. 19).

Aus der Perspektive der Anteilvorstellung beschreibt ein Bruch einen Zustand als Resultat einer Handlung (vgl. Wartha \& Güse, 2009, S. 263; Wartha, 2007, S. 64). Die Handlung, aus der der Zustand „, $\frac{3}{4}$ Kuchen“ als Ergebnis hervorgeht, kann auf (mindestens) zwei Weisen beschrieben werden:

(1) Ein Kuchen wird in vier gleichgroße Teile geteilt, drei dieser Teile werden betrachtet (Bruch als Teil eines Ganzen).

(2) Ein Kuchen wird verdreifacht und jeweils jeder vierte Teil jedes Kuchens genommen (Bruch als Teil mehrerer Ganzer).

Diese beiden möglichen Herstellungshandlungen sind äquivalent und führen zum gleichen Zustand „3 Kuchen“. Für einen tragfähigen Aufbau der Anteilvorstellung ist es jedoch wichtig, die Gleichwertigkeit dieser beiden Herstellungshandlungen als Verknüpfung der Bruchzahlaspekte Bruch als Teil eines Ganzen und Bruch als Teil mehrerer Ganzer herauszustellen (Padberg \& Wartha, 2017, S. 30; Postel, 1981, S. 29; vgl. auch Hefendehl-Hebeker, 1996).

Der statisch-abbildhafte Charakter der Anteilvorstellung kann auch anhand der weiteren oben aufgeführten Zahlaspekte verdeutlicht werden: 
(3) $\frac{1}{4} \mathrm{~km}$ ist der vierte Teil eines Kilometers, $\frac{3}{4} \mathrm{~km}$ ist das dreifache des vierten Teils eines Kilometers (Bruch als Maßzahl).

(4) $\frac{3}{4} h$ sind drei Viertelstunden (Bruch als Quasikardinalzahl).

(5) Jedes vierte Kaninchen ist schwarz und drei von vier Kaninchen sind weiß (Bruch als Quasiordinalzahl).

(6) Die Tankanzeige zeigt an, dass der Tank noch zu einem Viertel gefüllt ist (Bruch als Skalenwert).

Behr et al. (1983) beschreiben den Anteilaspekt als grundlegend für das Verständnis von weiteren Bruchzahlaspekten: „Partitioning and the part-whole subconstruct of rational numbers are basic to learning other subconstructs of rational numbers" (Behr et al. 1983, S.99). Aus diesem Grund wird in der Einführung von Bruchzahlen zumeist mit dem Ablesen und dem Einzeichnen von Anteilen in ikonischen Darstellungen gearbeitet (vgl. Padberg \& Wartha, 2017, S. 24 ff.). Die Darstellung eines Bruchs als Anteil ermöglicht einen direkten und anschaulichen Zugang zur Deutung und mentalen Repräsentation von Brüchen, die wesentliche Eigenschaften und Beziehungen eines Bruchs als Zustand enthält. Zum Erkennen des Anteils $\frac{3}{4}$ in einer ikonischen Abbildung (Abb. 2.4) müssen Lernende folgende Fragen verstehen und beantworten können (vgl. Padberg \& Wartha, 2017, S. 28):
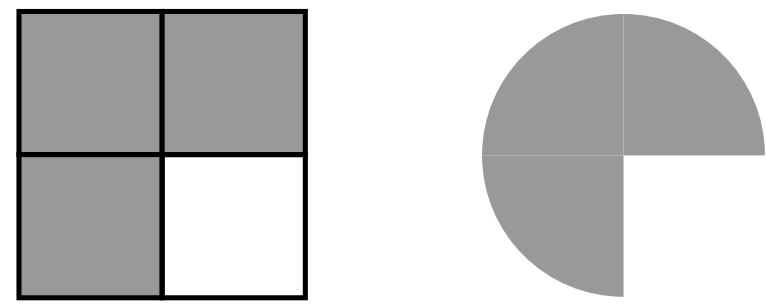

Abbildung 2.4 Ikonische Abbildung des Bruchs $\frac{3}{4}$ in einem Rechteck und einem Kreis

1. Was ist das Ganze?

2. In wie viele Teile ist das Ganze geteilt?

3. Sind die Teile jeweils gleich groß?

4. Wie groß ist jeder Teil bezogen auf das Ganze?

5. Wie viele Teile sind hier durch eine Färbung kenntlich gemacht worden?

Für einen verständigen Umgang mit Bruchzahlen ist es wichtig, dass die Lernenden geeignete Vorstellungen zum ,Bruchherstellungsakt“ (Postel, 1981, S. 22) bzw. 
zur Herstellungshandlung von Brüchen aufbauen, die es ihnen ermöglichen entsprechende Repräsentanten zu erkennen und mit dem Prozess ihrer Herstellung zu verbinden, sodass sie stets zwischen der ikonischen und enaktiven Ebene wechseln können. In diesem Zusammenhang bedarf es neben tragfähigen Vorstellungen von Brüchen als Anteil insbesondere einer dynamischen Sichtweise. Diese kommt vor allem in der Operatorvorstellung zum Tragen.

Grundvorstellung Bruch als Operator: Unter der Operatorvorstellung wird ein Bruch als eine Funktion aufgefasst, die ähnlich einer Skalarmultiplikation auf eine Größe wirkt (Vergnaud, 1988; Salle, 2015, S.131). Ein Bruchoperator ist als die Zusammenfassung zweier Teiloperatoren zu verstehen: Dem Zähleroperator, der als Multiplikator eine Größe (die Eingabe) vergrößert (streckt), und dem Nenneroperator, der als Divisor eine Eingabe verkleinert (staucht). Die Verkettung bzw. Hintereinanderausführung der beiden Teiloperatoren führt dazu, dass das Ergebnis (die Ausgabe) größer oder kleiner als die Eingabe sein kann (Oehl, 1970, S. 130f.; Postel, 1981, S. 22 ff.; Griesel, 1981c, S. 82). Die Anwendung eines Bruchoperators $\cdot \frac{m}{n}$ kann als Rechenanweisung interpretiert werden:

- „Dividiere durch $n$, multipliziere dann das Ergebnis mit $m$, bzw.

- multipliziere mit $m$, dividiere dann das Ergebnis durch $n$." (Postel, 1981, S. 24)

Die Hintereinanderausführung des Zähler- und Nenneroperators entspricht der Multiplikation mit einem Bruch. Diese multiplikative Beziehung wird sprachlich zumeist durch das Wort ,von“ zum Ausdruck gebracht: $\frac{3}{4}$ von $12 m=9 m$ (Wartha, 2007, S. 54; Padberg \& Wartha, 2017, S. 22). Der Bruchoperator $\frac{3}{4}$ wird auf die Größe $12 \mathrm{~m}$ angewendet und bildet $12 \mathrm{~m}$ auf $9 \mathrm{~m}$ ab.

Anschaulich beschreibt die Operatorvorstellung dynamisch das Verfahren der Bruchherstellung (vgl. auch Abb.2.2):

(1) „Man erhält eine $\frac{3}{4} m$ lange Strecke,

- indem man eine $1 \mathrm{~m}$ lange Strecke in 4 gleichlange Strecken zerlegt und dann 3 solcher Teilstrecken zusammensetzt, bzw.

- indem man eine $1 \mathrm{~m}$ lange Strecke auf den 4-ten Teil verkleinert (staucht) und anschließend das Ergebnis auf das 3-fache vergrößert (streckt). 
(2) Man erhält eine $\frac{3}{4} m$ lange Strecke,

- indem man drei $1 \mathrm{~m}$ lange Strecken aneinander legt und dann das Ergebnis in 4 gleichlange Strecken zerlegt, bzw.

- indem man eine $1 \mathrm{~m}$ lange Strecke auf das 3-fache vergrößert (streckt) und anschließend das Ergebnis auf den 4-ten Teil verkleinert (staucht)." (Postel, 1981, S.23)

Die beiden Beschreibungen des Bruchherstellungsverfahrens unterscheiden sich durch das Vertauschen der Zähler- und Nenneroperatoren, führen jedoch zu derselben Ausgabe bzw. demselben Ergebnis.

In Schulbüchern findet man die Operatorschreibweise vor allem in Form von Pfeildiagrammen (vgl. Abb. 2.5).

Man kann mit Brüchen auch Rechenanweisungen angeben.

Die Rechenanweisung davon $\frac{3}{4}$ bedeutet:

Zerlege das Ganze in vier gleich große Teile und nimm 3 davon.

oder

Dividiere eine Größe durch 4. Multipliziere dann das Zwischenergebnis mit 3.
Beispiel: Ein Zaun ist $60 \mathrm{~m}$ lang, $\frac{3}{4}$ davon ist bereits gestrichen. Wie viel $m$ sind das?

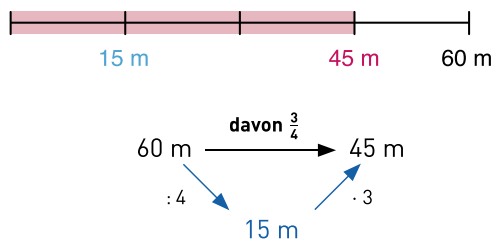

Lösung: $45 \mathrm{~m}$ des Zauns sind bereits gestrichen.

Abbildung 2.5 Schulbuchbeispiel zur Operatorschreibweise im Pfeildiagramm (vom Hofe, Humpert, Griesel \& Postel, 2012a, S. 184)

Als Vergleichsoperatoren (vgl. Malle, 2004, S.4) werden Brüche bzw. Bruchoperatoren häufig zum Vergleich zweier Mengen oder Größen verwendet. Vergnaud (1988) unterscheidet hierbei zwischen Fällen, in denen Bruchoperatoren relative Unterschiede zwischen zwei Mengen oder Größen beschreiben:

Inklusive Beziehungen beschreiben Teilungssituationen, in denen eine Größe mit einer Bezugsgröße derselben Art in Beziehung gesetzt werden, z. B. Ulf isst $\frac{3}{4}$ seiner Süßigkeiten.

Exklusive Beziehungen beschreiben Vergleiche zwischen zwei Größen, z.B. Ulf hat $\frac{3}{4}$ so viele Süßigkeiten gehortet wie Bernd. 
Freudenthal (1983) bezeichnet inklusive Beziehungen als direkten Vergleich und exklusive Beziehungen als indirekten Vergleich, bei dem zwei getrennte Objekte (Ulfs und Bernds Menge an Süßigkeiten) über ein drittes Objekt (Süßigkeiten als Maßeinheit) in Beziehung gesetzt werden. Die Unterscheidungen von Vergnaud (1988) und Freudenthal (1983) decken sich mit den Unterscheidungen, die Postel (1981, S. 17) unter dem Relationsaspekt von Bruchzahlen (siehe oben) beschreibt (vgl. auch Wartha, 2007, S. 55 f.).

Die Interpretation von Bruchzahlen als Vergleichsoperatoren kommt insbesondere dann zum Tragen, wenn (relative) Anteile von Größen betrachtet werden, z. B. $\frac{3}{4}$ von $10000 €$, und ist damit von besonderer Bedeutung für das Operieren mit Bruchzahlen.

Die Grundvorstellungen von Brüchen als Anteile und Operatoren stehen in engem Zusammenhang: Die Anteilvorstellung betont stärker die statische Komponente von Brüchen als Zustand, der das Ergebnis einer Handlung ist. Im Gegensatz dazu wird in der Operatorvorstellung stärker die dynamische Komponente in Hinsicht auf die (Herstellungs-) Handlung von Brüchen und Anteilen bzw. als Vorschrift zur Änderung von Größen herausgestellt. Für einen verständigen Umgang mit Bruchzahlen und das rechnerische Operieren mit ihnen sind beide Grundvorstellungen grundsätzlich.

Drei Grundaufgaben: In Hinsicht auf die Anwendung in Sachkontexten werden durch Brüche vor allem Zusammenhänge zwischen Teil, Anteil und Ganzem beschrieben. Hierbei können drei „Grundaufgaben“ (Padberg \& Wartha, 2017, S. 34 ff.; Postel, 1981, S.26ff.) bzw. „Konstellationen“ (Schink, 2013, S. 54 ff.) unterschieden werden ${ }^{8}$ :

(1) Bestimmen eines Teils einer Größe: Das Ganze und der Anteil (Operator) sind gegeben, der Teil soll bestimmt werden, z. B.: Jans Familie besitzt einen Bauernhof mit einer $96 h a$ große landwirtschaftlichen Nutzfläche. Auf $\frac{5}{8}$ dieser Fläche wird Getreide angebaut. Wie viel ha Land sind das?“

(2) Bestimmen des Ganzen: Der Anteil (Operator) und der Teil sind gegeben, das Ganze soll bestimmt werden, z. B.: Patrick will sich ein Fahrrad kaufen. Er hat $240 €$ gespart. Patrick sagt: „Ich habe schon $\frac{2}{3}$ des Kaufpreises zusammen.“ Wie teuer ist das Fahrrad?

\footnotetext{
${ }^{8}$ Eine ähnliche Klassifizierung wird üblicherweise in der Prozent- und Zinsrechnung eingeführt (vgl. Hafner, 2012).
} 
(3) Bestimmen von Anteilen: Das Ganze und der Teil sind gegeben, der Anteil (Operator) soll bestimmt werden, z. B.: Sarah bekommt monatlich $15 €$ Taschengeld. Davon spart sie $4 €$. Welchen Anteil des Taschengeldes spart sie?

Obgleich diese drei Grundaufgaben in Schulbüchern (vgl. z. B. vom Hofe, Humpert, Griesel \& Postel 2012b, S. 49 ff.) häufig als eigenständiger Unterrichtsinhalt mit dazugehörigen Lösungsverfahren behandelt wird, so zeigen sich in der Empirie (vgl. Schink, 2013; Tunc-Pekkan, 2015) deutliche Schwierigkeiten von Lernenden bei der Bearbeitung von Aufgaben, in denen der Anteil bestimmt oder von einem Teil auf das Ganze geschlossen werden soll. Schink (2013) weist darauf hin, dass Lernende in Hinsicht auf einen flexiblen Umgang mit Brüchen die Beziehungen zwischen den drei Grundaufgaben bzw. die Beziehungen zwischen Teil, Anteil und Ganzem erschließen und durchdringen müssen: „Wer flexibel mit Brüchen umgehen will, der muss auch Einsichten in Strukturen gewinnen und diese flexibel nutzen“ (Schink, 2013, S. 62) (Abb. 2.6).

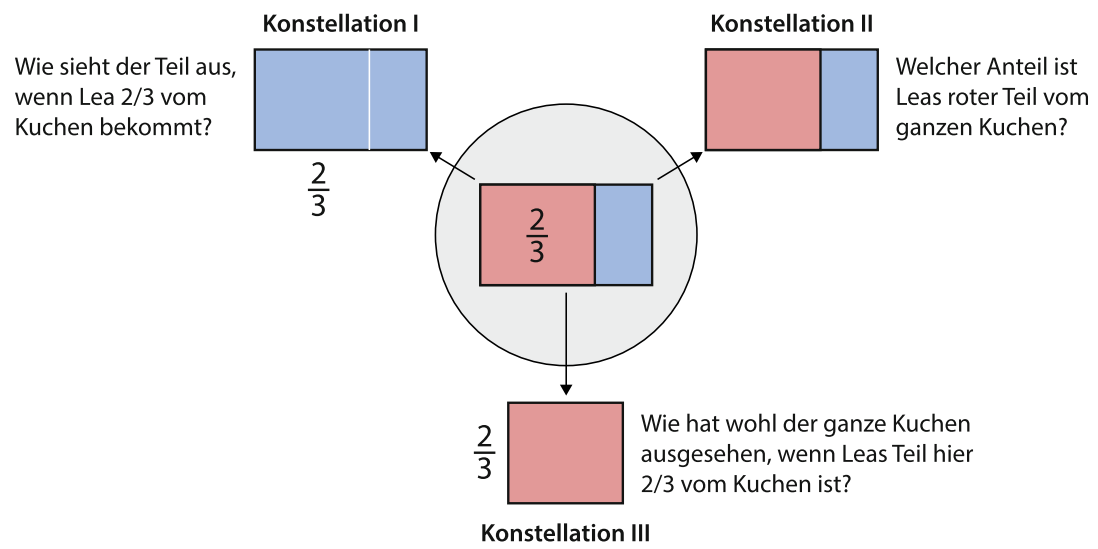

Abbildung 2.6 Drei Grundaufgaben (bzw. Konstellationen) zum Bruch als Teil eines Ganzen (Schink, 2013, S. 56)

Insbesondere die Inversion des Bruchherstellungsverfahrens zum Schluss von einem Teil auf das zugrundeliegende Ganze („Reversibilität“ bei Ramful, 2014) stellt Lernende vor Schwierigkeiten. Während dieser Schluss speziell bei Stammbrüchen $\frac{1}{n}$ lediglich eines $n$-fachen Vervielfachens des Teils entspricht, muss bei echten Brüchen $\frac{m}{n}$ mit $m>1$ der Teil zunächst in $m$ gleich große Teilstücke zerlegt werden, bevor diese durch eine $n$-fache Vervielfachung das Ganze ergeben. 


\subsubsection{Grundvorstellungen zum Operieren mit Bruchzahlen}

Neben den dargestellten Bruchzahlaspekten und der Ausbildung entsprechender Grundvorstellungen zu Bruchzahlen selbst, erfordert auch das verständige Operieren mit Bruchzahlen einer Vorstellungsgrundlage. In Bezug auf den inhaltlichen Schwerpunkt dieser Arbeit werden im Folgenden Vorstellungsgrundlagen für das Übersetzen zwischen verschiedenen Darstellungen, das Erweitern und Kürzen sowie den Größenvergleichen von Brüchen und Bruchzahlen dargestellt.

Übersetzen zwischen Bruchdarstellungen: Der Wechsel zwischen verschiedenen Darstellungen von Brüchen dient im Wesentlichen der Vernetzung von Bruchzahlvorstellungen und verschiedenen Bruchzahlaspekten, sodass die Herausbildung von ,isolierten Inselvorstellungen“ vermieden wird (vgl. Behr, Post, Harel \& Lesh, 1993; Kieren, 1993). Brüche sollen in unterschiedlichen Anwendungssituationen erkannt und benutzt werden können. Hierbei muss vor allem auf Grundvorstellungen vom Bruch als Anteil und als Operator zurückgegriffen werden und eine Verbindung zum jeweiligen Bruchzahlaspekt in der Darstellung hergestellt werden.

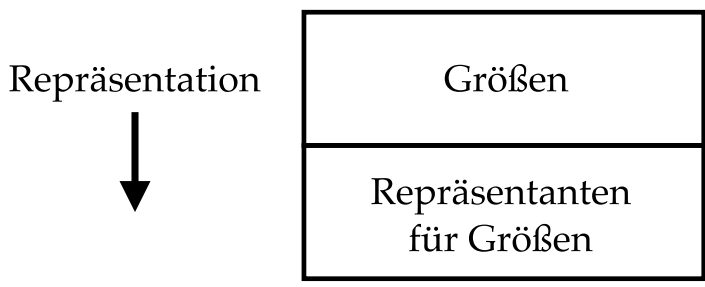

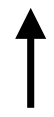

Abstraktion

Abbildung 2.7 Beziehungen zwischen Größen und Repräsentanten (Abb. nach Griesel, 1973, S. 14)
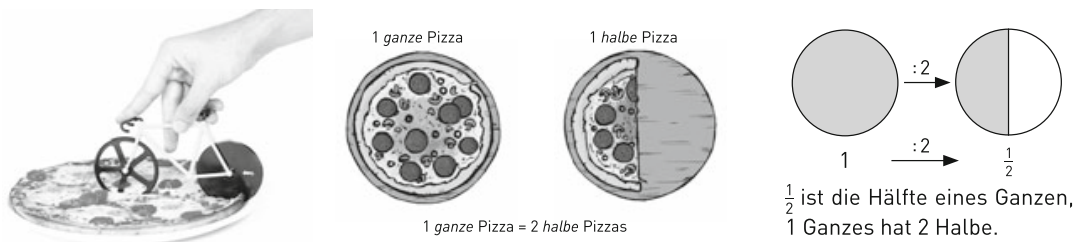

Abbildung 2.8 Übertragung der ikonischen Darstellung des Bruchs $\frac{1}{2}$ von der Realisantenebene (links) auf die Repräsentantenebene (rechts). (Abb. aus vom Hofe, Humpert, Griesel \& Postel, 2012a, S. 170f.) 
Bildliche Darstellungen dienen neben der Illustration von Bruchsituationen vor allem als Grundlage für den Aufbau mentaler Repräsentationen. Zumeist werden Brüche anhand von Auf- und Verteilungskontexten in Bezug auf Objekte der alltäglichen Erfahrungswelt, wie. z. B. dem gerechten Aufteilen einer Pizza, eingeführt. Das Ziel ist es hierbei, den Prozess der Herstellung von Brüchen an erfahrbare Handlungen zu knüpfen, die im weiteren Lernverlauf kontinuierlich abstrahiert werden. Die konkret-gegenständlichen Repräsentanten einer Größe werden aus ihrem gegenständlichen Kontext gelöst und auf die Ebene der Repräsentanten von Größen, z. B. Kreise und Rechtecke, übertragen, wie es in den Abb. 2.8 und 2.7 illustriert ist. Mehr oder weniger abstrakte Repräsentationen ersetzen konkrete Repräsentanten und bilden die Grundlage für mentale Repräsentationen von Bruchzahlen $\frac{m}{n}$ und ihrer Herstellung. Die verwendeten Repräsentanten sollen dabei so gewählt werden, dass sie nicht lediglich eine ,,illustrierende Funktion“ (Padberg \& Wartha, 2017, S. 32) haben, sondern eine möglichst tragfähige und sachadäquate Abbildung einer Situation ermöglichen:

„Eine Größe, wie z. B. $\frac{3}{4} m$ läßt sich als solche nicht vorstellen, wohl aber ihre Repräsentanten, in unserem Fall Strecken der Länge $\frac{3}{4} m$. Insbesondere für ein inhaltliches Denken und eine intuitive Begründung von mathematischen Zusammenhängen sind solche Vorstellungen unerläßlich“. (Griesel, 1981a, S.9)

Für das Übersetzen zwischen Bruchdarstellungen muss zwischen einem (1) Wechsel der Repräsentationsebene und einem (2) Wechsel innerhalb einer Repräsentationsebene unterschieden werden:

(1) Wechsel zwischen Repräsentationsebenen betreffen zumeist das Übersetzen zwischen ikonischen und symbolischen Bruchdarstellungen. Beim Ablesen von Brüchen aus ikonischen Repräsentationen kommt vor allem die Anteilvorstellung zum Tragen, während beim bildlichen Darstellen eines symbolisch vorgegebenen Bruchs vor allem die Operatorvorstellung mit Bezug auf Herstellungshandlung als Teilen des Ganzen in gleich große Teile und Markieren des entsprechenden Anteils zum Tragen kommt. Das Ziel von Repräsentationswechseln zwischen verschiedenen Repräsentationsebenen entspricht dem Wechsel zwischen verschiedenen Deutungen bzw. der Überführung auf die anschauliche Bild-/Handlungsebene oder die abstrakte symbolische Zahlenebene.

(2) Der Wechsel innerhalb einer Repräsentationsebene betrifft einerseits Umwandlungen von unechten Brüchen in gemischte oder natürliche Zahlen, z.B. $\frac{3}{2}=1 \frac{1}{2}$, auf symbolischer Ebene und andererseits der Repräsentation einer 
Bruchsituation in verschiedenen ikonischen Darstellungen. Der Wechsel zwischen verschiedenen ikonischen Darstellungen (vgl. Abb. 2.9) soll es den Lernenden ermöglichen unterschiedliche Begriffsaspekte miteinander zu verknüpfen und allgemein flexiblere Anschauungsgrundlagen von Brüchen aufzubauen.

Der Wechsel zwischen verschiedenen Darstellungen kann als Koordination von Wissen beschrieben werden, bei der Wissen über die Sachstruktur, d.h. über das mathematische Objekt mit seinen Eigenschaften und Relationen, und das Wissen über die Eigenschaften eines Repräsentationssystems und dessen Verbindungen zum mathematischen Inhalt miteinander koordiniert werden. Dies erfordert einerseits das Herauslösen des mathematischen Objekts aus der initialen Repräsentation (vgl. „Dissociation“ bei Duval, 2006, S. 124) und der Einbettung in eine andere Repräsentation, was eine Reorganisation des Wissens über die Repräsentation erfordert (vgl. Superfine, Canty \& Marshall, 2009; Novick, Hurley \& Francis, 1999). In diesem Prozess kommt der Anteil- und Operatorvorstellung eine tragende Rolle zu.
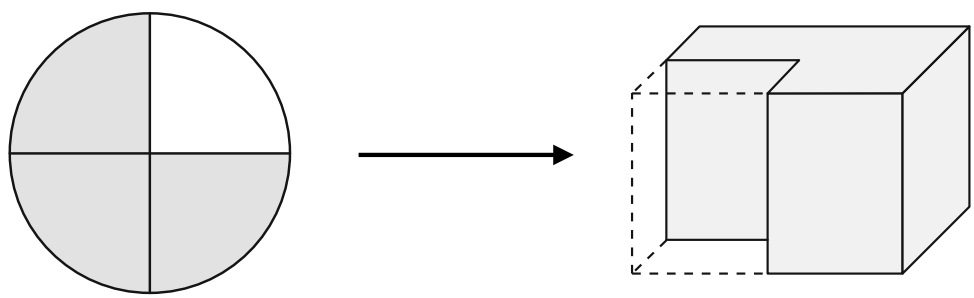

Abbildung 2.9 Ikonische Darstellung des Bruchs $\frac{3}{4}$ in Kreisrepräsentation und dreidimensionalen Quaderrepräsentation (Abb. aus vom Hofe, Humpert, Griesel \& Postel, 2012a, S. $173 \mathrm{f}$.
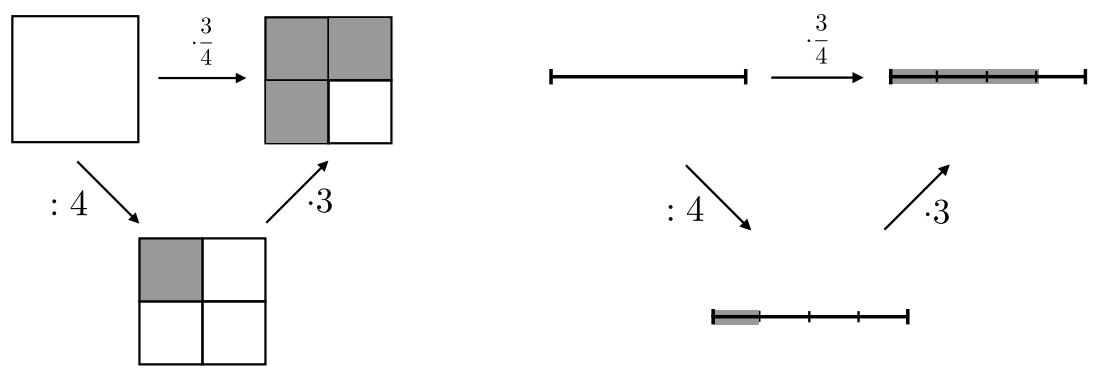

Abbildung 2.10 Übertragung der ikonischen Darstellung des Bruchs $\frac{3}{4}$ in einem Quadrat (links) auf die ikonische Darstellung an einer Strecke (rechts) 
Zum Übertragen der ikonischen Darstellung des Bruchs $\frac{3}{4}$ in Abb. 2.10 müssen die Struktureigenschaften der Darstellung des Bruchs in einer quadratischen Fläche zunächst von dieser gelöst werden, um sie auf die Darstellung in einer Strecke zu übertragen. Die Struktureigenschaften der Darstellung des Bruchs in einer quadratischen Fläche werden durch die Herstellungshandlung beschrieben. Die ganze Fläche wird zunächst in vier kongruente Teilflächen geteilt, von denen eine Teilfläche bzw. $\frac{1}{4}$ der ganzen Fläche gefärbt ist. Dieser vierte Teil wird in einem weiteren Schritt verdreifacht, sodass insgesamt drei der vier Teilflächen gefärbt sind. Zur Darstellung des Bruchs in einer Strecke ist die Herstellungshandlung bzw. die Darstellungshandlung isomorph zur Darstellung in einer quadratischen Fläche: Die ganze Strecke wird zunächst in vier gleich große Teilstücke zerlegt, von denen ein Teilstück $\frac{1}{4}$ der Strecke ist. Dieser Teil wird in der Folge verdreifacht und somit werden insgesamt drei der vier Teilstücke eingefärbt. Die graue Färbung der Strecke repräsentiert die neue Länge der Strecke.

Für dieses Beispiel muss entsprechend die Herstellungs- bzw. Darstellungshandlung des Bruchs aus der Darstellung in einer quadratischen Fläche dissoziiert, d.h. herausgelöst werden. Zur Übertragung auf die Darstellung an einer Strecke muss diese jedoch an die Repräsentationseigenschaften der Strecke angepasst werden. Eine Strecke wird im Gegensatz zu einer Fläche nicht in kongruente Teilflächen, sondern in gleich lange Teilstrecken zerlegt. Die zugrundeliegende Größe ist somit kein Flächeninhalt, sondern eine Länge. Obgleich die Zeichenhandlungen strukturell isomorph sind, sind sie nicht identisch, sondern spezifisch für die einer neuen Darstellung zugrundeliegenden Eigenschaften.

Da eine Darstellung zumeist nur vereinzelte Aspekte von Brüchen repräsentiert, ist es in Hinsicht auf den Aufbau vernetzter und flexibler Bruchzahlvorstellungen von Bedeutung verschiedene Darstellungen zu verwenden und miteinander in Beziehung zu setzen. Damit Lernende von einer Vielfalt von Darstellungen profitieren können ist es jedoch notwendig, dass sie einzelne Darstellungen adäquat interpretieren können und verschiedene Darstellungen miteinander in Beziehung setzen. Geschieht dies nicht, so kann der Einsatz unterschiedlicher Darstellungen eine Hürde für den Lernprozess darstellen, da Lernende anhand von unbekannten Darstellungen nur schwer etwas über unbekannte konzeptuelle Inhalte und Zusammenhänge lernen können (Rau \& Matthews, 2017, S. 542).

Erweitern und Kürzen von Brüchen: Das Erweitern und Kürzen ist ein erster anschaulicher Zugang zur Äquivalenz von Brüchen. Durch das Kürzen oder Erweitern werden Repräsentanten einer Bruchzahl auf symbolischer Ebene ineinander überführt. Grundlegend für das Verständnis ist hierbei, dass der Ausgangsbruch äquivalent zum gekürzten oder erweiterten Bruch ist: $\frac{m}{n}=\frac{m \cdot k}{n \cdot k}$ für alle $k \in \mathbb{N}$. 
Eine Bruchzahl kann auf diese Weise durch (unendlich) viele Brüche beschrieben werden. Dieses Wissen ist eine grundlegende Komponente von Grundvorstellungen bzw. kognitiven Repräsentationen des Bruchzahlbegriffs (vgl. Ni, 2001, S. 412). Zudem steht die Einsicht in die Äquivalenz quotientengleicher Paare in enger Beziehung zum proportionalen Denken und ist nicht nur für die Ausbildung adäquater und tragfähiger Vorstellungen von Bruchzahlen und Bruchoperationen wichtig, sondern wird im Allgemeinen als eine zentrale Idee mathematischen Denkens beschrieben (vgl. Wartha, 2007, S.67; Hunting, 1984, S. 32).

In Bezug auf den Aufbau vernetzter und tragfähiger Bruchzahlvorstellungen können Lernende durch das Erweitern und Kürzen von Brüchen im Zusammenhang mit verschiedenen Bruchzahlaspekten erste Erfahrungen mit äquivalenten Brüchen sammeln:

„Verschiedene Brüche (z.B. $\frac{3}{4}$ und $\left.\frac{6}{8}\right)$

- bezeichnen bei derselben Maßeinheit (z. B. $\mathrm{cm}^{2}$ ) dieselbe Größe, z. B. $\frac{3}{4} \mathrm{~cm}^{2}=\frac{6}{8} \mathrm{~cm}^{2}$ (Maßzahlaspekt),

- geben denselben Bruchteil an (Relationsaspekt),

- bewirken bei Bruchoperatoren dasselbe, wie z. B. $\cdot \frac{3}{4}=\cdot \frac{6}{8}$ (Operatoraspekt),

- geben denselben Punkt auf dem Zahlenstrahl an (Skalenwertaspekt)“. (Postel, 1981, S. 28)

Hinsichtlich einer systematischen Erarbeitung des Erweiterns und Kürzens stellt die Interpretation des Verfeinerns und Vergröberns einer Einteilung eine zentrale Grundvorstellung dar. Beim Verfeinern wird die Einteilung einer ikonischen Bruchzahldarstellung so verändert, dass jeder Teil der ursprünglichen Einteilung in weitere gleich große Teile unterteilt wird. Wird zum Beispiel jeder Teil einer ursprünglichen Einteilung in zwei gleich große Teile geteilt, hat die neue Einteilung insgesamt doppelt so viele Teile. Dies entspricht dem Erweitern des Bruches mit dem Faktor $2 \mathrm{zu} \frac{6}{8}$. Werden alle Teile der neuen Einteilung ein weiteres Mal in zwei gleich große Teile geteilt, hat die neue Einteilung im Vergleich zur ursprünglichen Einteilung viermal so viele Teile und die ikonische Darstellung repräsentiert den Bruch $\frac{12}{16}$ (vgl. Abb. 2.11). Auf symbolischer Ebene entspricht das Verfeinern einer Einteilung eines Bruchs $\frac{m}{n}$ der Multiplikation von Zähler und Nenner mit einem gemeinsamen Faktor $k: \frac{m}{n}=\frac{m \cdot k}{n \cdot k}$ für alle $k \in \mathbb{N}$.

Das Vergröbern einer Einteilung kann als Umkehrung des Verfeinerns interpretiert werden. Statt jeden Teil der Ursprungseinteilung in weitere gleich große Teile zu teilen, werden Teile der ursprünglichen Einteilung zusammengefügt, sodass sie 

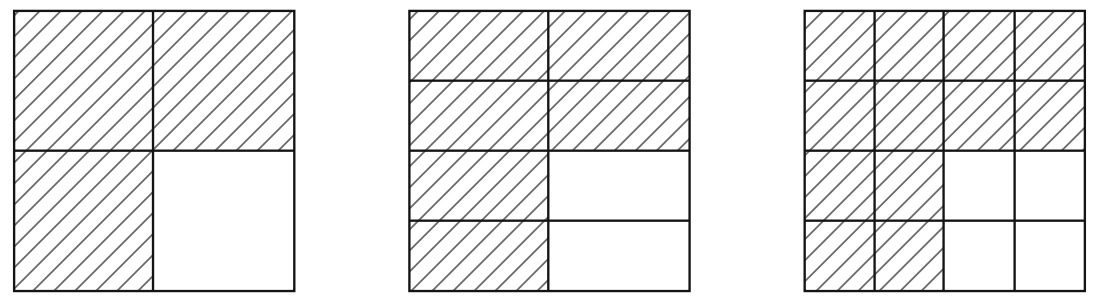

Abbildung 2.11 Verfeinern der Einteilung der ikonischen Darstellung des Bruchs $\frac{3}{4}$ (Abb. aus Padberg \& Wartha, 2017, S. 44)

einen neuen, größeren Teil ergeben. Beim Vergröbern der Einteilung des Bruchs $\frac{8}{12}$ entsteht durch Zusammenfügen von je zwei Teilen zu einem neuen Teil eine neue Einteilung mit insgesamt sechs Teilen, sodass die neue Darstellung den Bruch $\frac{4}{6}$ repräsentiert. Eine weitere Vergröberung der Einteilung, bei der wiederum zwei Teile zu einem neuen größeren Teil zusammengefügt werden, ergibt die Einteilung des Bruchs $\frac{2}{3}$ (vgl. Abb. 2.12). Auf symbolischer Ebene entspricht das Vergröbern einer Einteilung eines Bruchs $\frac{m}{n}$ der Division von Zähler und Nenner mit der gleichen Zahl $k: \frac{m}{n}=\frac{m: k}{n: k}$ für alle gemeinsamen Teiler $k$ von $m, n \in \mathbb{N}$.

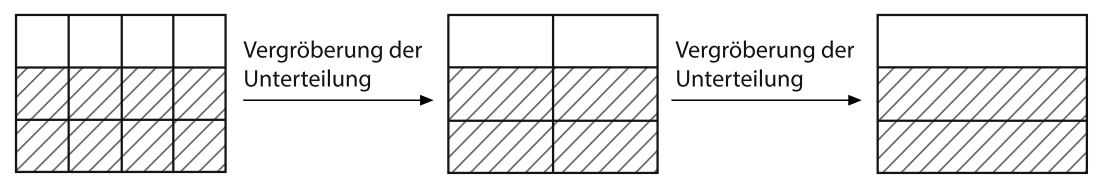

Abbildung 2.12 Vergröbern der Einteilung der ikonischen Darstellung des Bruchs $\frac{8}{12}$ (Abb. aus Padberg \& Wartha, 2017, S. 49)

Während dem Verfeinern der Einteilung von Brüchen keine Grenzen gesetzt sind und eine Einteilung in beliebig viele kleinere Teile geteilt werden kann, ist das Vergröbern einer Einteilung auf gemeinsame Teiler von Zähler und Nenner beschränkt. Sofern der Zähler und Nenner eines Bruchs teilerfremd sind, ist kein weiteres Kürzen bzw. Vergröbern der Einteilung möglich.

Malle (2004) bemerkt, dass in einer ersten Phase der Einführung von Bruchzahlen auf die Begriffe „Erweitern und Kürzen“ verzichtet werden solle, da diese eine „regelhafte Technik“ bezeichnen: 
„Wichtiger ist zunächst, dass der Prozess des Umwandelns einer Bruchdarstellung in eine andere mit der Vorstellung des Verfeinerns bzw. Vergröberns verbunden wird und dabei eingesehen wird, dass man eine Bruchzahl auf unendlich viele Arten durch Brüche darstellen kann.“ (Malle, 2004, S.6)

Aus diesem Grund kommt dem Vernetzen von Handlungen auf enaktiver, ikonischer und symbolischer Ebene eine wichtige Rolle zu. Das Ziel ist,

„daß Schüler durch die Arbeit mit konkreten Modellen anschauliche Vorstellungen über das Erweitern und Kürzen erwerben müssen, daß dann die konkrete Arbeit mit Modellen und die Ziffernmanipulationen in Beziehung gesetzt werden müssen und daß schließlich die Fähigkeit entwickelt werden muß, die entsprechenden Algorithmen konsistent und korrekt auf Grundlage einer gut entwickelten, modellunabhängigen Idee anzuwenden.“ (Payne, 1986, S. 56)

Die Grundvorstellung von Verfeinern und Vergröbern einer Einteilung baut vornehmlich auf dem Anteil- und Maßzahlaspekt von Brüchen auf (Postel, 1981, S. 28 ff.; Wartha, 2007, S. 67 f.). Ein weiteres Erklärungsmodell beschreibt Postel (1981) über die Grundvorstellung vom Bruch als Operator. Die Grundidee ist hierbei nachzuweisen, dass die Anwendung äquivalenter Bruchoperatoren dieselbe Wirkung auf eine Größe haben. So kann auf symbolischer Ebene über den Vergleich von Operatortabellen beispielgebunden begründet werden, dass die Operatoren $\cdot \frac{3}{4}$ und $\cdot \frac{6}{8}$ auf dieselbe Eingabe (Größe) angewendet dieselbe Ausgabe haben (vgl. Abb. 2.13).
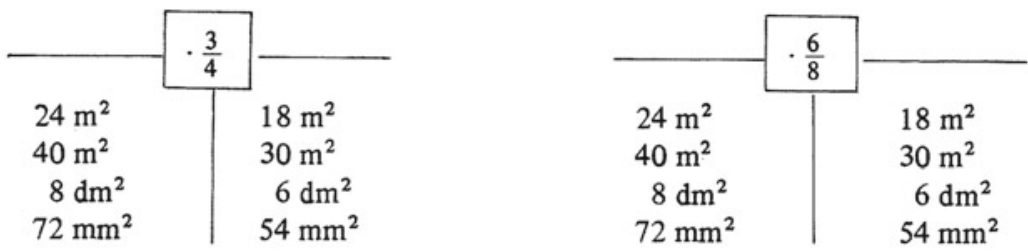

Also: $\frac{3}{4}=\frac{6}{8}$

Abbildung 2.13 Vergleich der Wirkung der Operatoren $\cdot \frac{3}{4}$ und $\cdot \frac{6}{8}$ von Operatortabellen (Abb. aus Postel, 1981, S. 28)

Auf ikonischer Ebene kann die Äquivalenz der beiden Operatoren über eine Verknüpfung der Herstellungshandlungen herausgestellt werden. Hierbei wird deutlich, dass der Operator $\cdot \frac{3}{4}$ durch die Multiplikation von Zähler und Nenner mit dem Fak- 
tor 2 in den Operator $\cdot \frac{6}{8}$ überführt werden kann. Die Multiplikation von Zähler und Nenner mit dem Faktor 2 bewirkt, dass das Ganze in doppelt so viele Teile eingeteilt wird und schließlich die doppelte Anzahl von Teilen markiert werden, wodurch der entstehende Anteil gleich ist (vgl. Abb. 2.14).
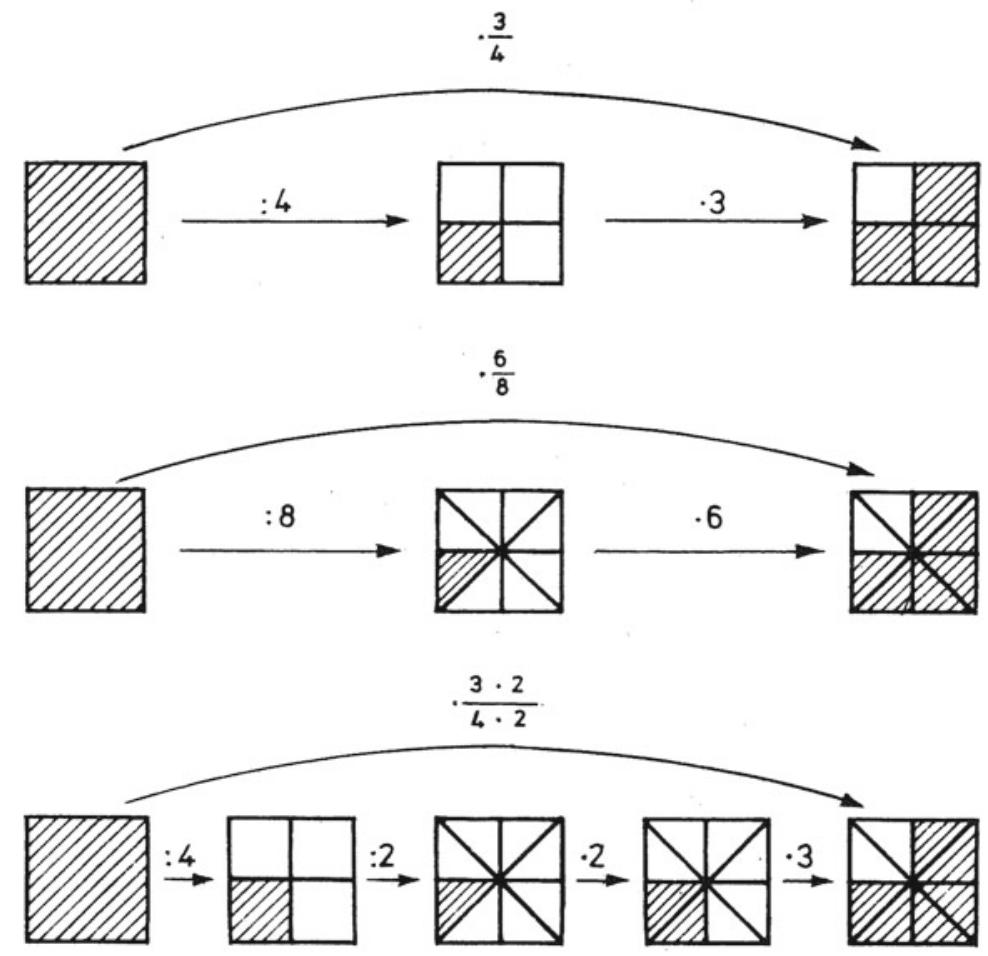

Abbildung 2.14 Vergleich der Wirkung der Operatoren $\cdot \frac{3}{4}$ und $\cdot \frac{6}{8}$ auf ikonischer Ebene (Abb. aus Postel, 1981, S. 29)

Die Einsicht, dass ein Bruch $\frac{m}{n}$ durch Erweitern und Kürzen in unendlich viele äquivalente Bruchdarstellungen umgewandelt werden kann und somit unendlich viele Repräsentanten hat, ist ein grundlegender Schritt in der Loslösung der Sicht von Brüchen als Anteile von konkreten Repräsentationsobjekten und der Auffassung von Brüchen als Zahlen, die auf der Zahlengerade angeordnet werden und mit denen gerechnet werden kann. Ein inhaltliches Verständnis der Äquivalenz von Brüchen 
ist somit grundlegend für die Einführung von Ordnungsrelationen und eine wichtige Voraussetzung für das spätere Addieren und Subtrahieren von Bruchzahlen.

Größenvergleich von Brüchen und Bruchzahlen: Das Größenvergleich von Brüchen ist ein wesentlicher Schritt in der Entwicklung von Grundvorstellungen von Brüchen als anschauliche Größen zu einem Verständnis von Bruchzahlen als Punkte auf der Zahlengerade.

Postel (1981, S. 30 f.) beschreibt drei Vorstellungen, die Lernende mit dem Größenvergleich von Brüchen verbinden sollen:

(1) „Größenaspekt: Zur kleineren Bruchzahl gehört eine kleinere Größe.

Beispiel: In einem Becher befinden sich $\frac{3}{4} l$ Sahne, in einem anderen Becher $\frac{5}{8} l$ Sahne. In welchem Becher ist weniger Sahne?

(2) Relations- bzw. Operatoraspekt: Der zur kleineren Bruchzahl gehörende Operator bewirkt weniger als der zur größeren Bruchzahl gehörende Operator bzw. zur kleineren Bruchzahl gehört der kleinere Bruchteil.

Beispiel: Zwei Kopiergeräte stellen unterschiedliche Kopien her: Bei Gerät A beträgt die Länge eines Gegenstandes im Bild $\frac{5}{8}$ der ursprünglichen Länge. Bei Gerät B beträgt sie $\frac{7}{8}$ der ursprünglichen Länge. Welches Gerät stellt die kleineren Bilder her? [...]

(3) Skalenwertaspekt: Die kleinere Bruchzahl liegt links von der größeren Bruchzahl auf dem Zahlenstrahl.

Beispiel: Der Wasserstand betrug morgens $1 \frac{3}{5} \mathrm{~m}$, abends $1 \frac{1}{2} \mathrm{~m}$. Wann wurde der niedrigere Wasserstand angezeigt?“ (Postel, 1981, S. 30 f.).

In diesen drei Beispielen ist zu erkennen, dass der Größenvergleich von Brüchen und Bruchzahlen auf Grundlage der Grundvorstellungen Bruch als Anteil und Bruch als Operator vorgenommen werden können, die im weiteren Verlauf als Erklärungsmuster (vgl. Wartha, 2007, S. 30 ff.) erläutert werden. Zudem ist in Postels Beschreibung des Größenvergleichs unter dem Skalenwertaspekt die Übertragung zur Anordnung von Brüchen auf der Zahlengerade angedeutet.

Mit Bezug auf die Grundvorstellung Bruch als Anteil können Brüche als Anteile von Größen anschaulich direkt miteinander verglichen werden (vgl. Abb. 2.15). Anhand der Darstellung in einer ikonischen Repräsentation wie z. B. in einem Quadrat kann unmittelbar erkannt werden, dass der Bruch $\frac{5}{8}$ kleiner ist als der Bruch $\frac{7}{8}$, da in der Darstellung des Bruchs $\frac{7}{8}$ eine größere Fläche gefärbt ist als in der Darstellung des Bruchs $\frac{5}{8}$. Entsprechend ist zu erkennen, dass zum kleineren Bruch eine kleinere Größe gehört. Dies setzt voraus, dass die Ausgangsflächen (die Qua- 
drate) jeweils gleich groß sind und in acht gleich große Teile geteilt wurden. Der Vergleich von gleichnamigen Brüchen über ihre ikonische Darstellungen ist ohne Schwierigkeiten generalisierbar, da dieses Verfahren sich auf andere Größen und Darstellungen übertragen lässt.

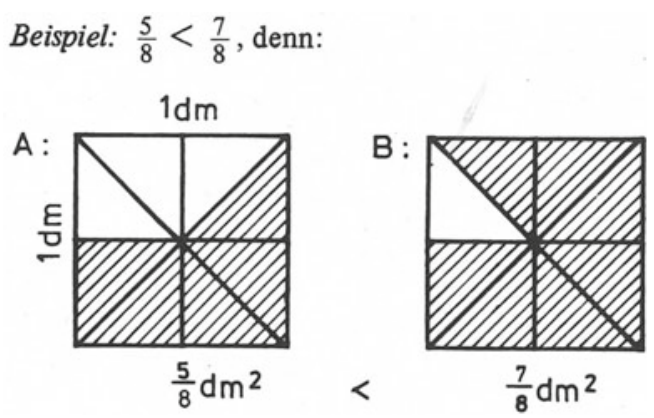

Abbildung 2.15 Größenvergleich zweier Brüche mit gleichem Nenner auf ikonischer Ebene (Abb. aus Postel, 1981, S. 31)

Auf Grundlage der Grundvorstellung Bruch als Operator kann auf zwei Wegen ein Größenvergleich zwischen zwei Brüchen vorgenommen werden: Über den Vergleich von Operatortabellen oder durch die ikonische Darstellung der Bruchherstellung:
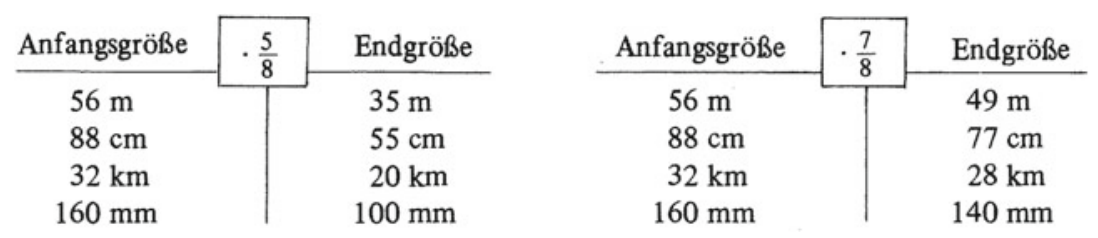

Abbildung 2.16 Größenvergleich zweier Brüche mit Operatortabellen (Abb.aus Postel, 1981, S. 32)

Wenn Brüche als Operatoren interpretiert werden, bewirkt der kleinere Bruchoperator eine geringere Änderung als der größere Bruchoperator (vgl. Abb. 2.16). Bei gleicher Ausgangsgröße ist zu erkennen, dass der Operator $\frac{5}{8}$ jeweils eine kleinere Endgröße ausgibt als der Operator $\frac{7}{8}$, demnach ist der Bruch $\frac{5}{8}$ kleiner als der Bruch $\frac{7}{8}$. 
Analoge Einsichten ermöglicht der Vergleich der Bruchherstellungsverfahren der zu vergleichenden Brüche (vgl. Abb. 2.17). In beiden Fällen wird die gleiche Ausgangsgröße in acht gleich große Teile zerlegt. Beim Bruch $\frac{5}{8}$ werden im zweiten Schritt weniger Teile zusammengefügt als beim Bruch $\frac{7}{8}$. Diese Vergleichsmethode kann auch auf das Verkleinern und Vergrößern von Repräsentanten übertragen werden. Die Argumentation lautet dann: „In beiden Fällen wird im gleichen Maße verkleinert, nämlich auf den 8 -ten Teil. Bei $\cdot \frac{5}{8}$ wird anschließend weniger stark vergrößert als bei $\cdot \frac{7}{8}$ “ (Postel, 1981, S.33).

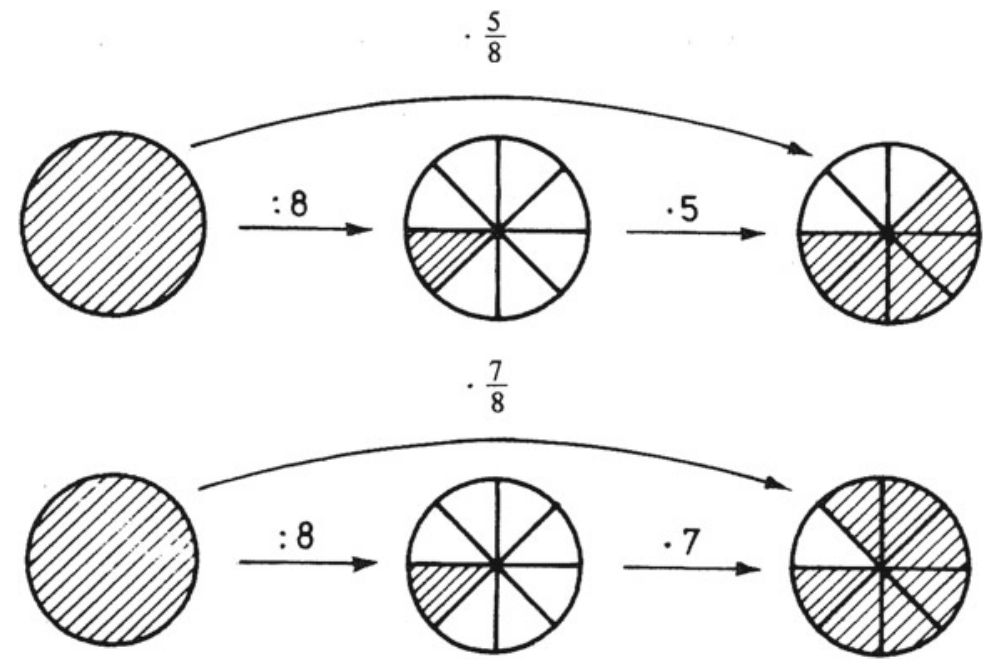

Abbildung 2.17 Größenvergleich zweier Brüche über ihre Herstellung (Abb.aus Postel, 1981, S. 32)

Im Kern des Größenvergleichs von Brüchen auf ikonischer Ebene steht die gleiche Einteilung der zu vergleichenden Größen und Repräsentanten. Das bedeutet, dass die jeweiligen Ausgangsgrößen gleich groß sind und auch die Teile der Einteilung die gleiche Größe haben. Nur in diesem Fall kann die Ordnungsbeziehung aus den natürlichen Zahlen auf den Vergleich von Brüchen übertragen und argumentiert werden: $\frac{5}{8}<\frac{7}{8}$, weil 5 kleiner ist als 7 . Entsprechend können nur gleichnamige Brüche auf diesen Wegen direkt miteinander verglichen werden. Sofern diese Voraussetzung nicht gegeben ist, ist es notwendig die Einteilung zunächst zu vereinheitlichen. 
Ein Vereinheitlichen der Einteilung als anschauliche Vorstellung für das Erweitern und Kürzen der zu vergleichenden Brüche auf einen gemeinsamen Hauptnenner kann über das ,Übereinanderlegen“ von Einteilungen dargestellt werden (Padberg \& Wartha, 2017, S. 60): Zum Beispiel können die Brüche $\frac{5}{8}$ und $\frac{2}{3}$ der Größe nach verglichen werden, indem beide Brüche zunächst in einem gleich großen Rechteck eingetragen werden. Dabei wird das Rechteck für den Bruch $\frac{5}{8}$ horizontal und für den Bruch $\frac{2}{3}$ vertikal unterteilt. Durch übereinanderlegen der beiden Rechtecke erhält man eine gemeinsame Einteilung in $3 \cdot 8=24$ gleiche Teile. Nun können die Anzahlen der markierten Teile verglichen werden und festgestellt werden, dass 15 Teile weniger als 16 Teile sind und folglich $\frac{5}{8}$ kleiner als $\frac{2}{3}$ ist (vgl. Abb. 2.18).

Welcher der Brüche $\frac{5}{8}$ und $\frac{2}{3}$ ist größer? Begründe.

\section{Lösung im Rechteckmodell:}
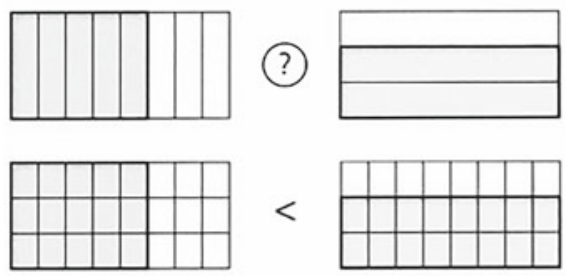

16 Teilstücke sind mehr als 15 ebenso große Teilstücke.

Abbildung 2.18 Größenvergleich durch Übereinanderlegen von Einteilungen (Abb.aus Padberg \& Wartha, 2017, S. 60)

Auf symbolischer Ebene beschreiben Behr et al. (1984) neben dem Vergleich von Brüchen über gleichnamige Nenner drei weitere Strategien (vgl. auch Clarke \& Roche, 2009):

Vergleich über gleichnamige Zähler: Beim Vergleich von Brüchen mit gleichem Zähler kann der Größenvergleich durch Vergleich von Stammbrüchen vorgenommen werden. Für den Größenvergleich der Brüche $\frac{2}{5}$ und $\frac{2}{3}$ bedeutet der gleiche Zähler, dass die gleiche Anzahl von Teilen betrachtet wird. Entsprechend muss die Größe der Teile verglichen werden. Wird beispielsweise eine Strecke der Länge $1 \mathrm{~m}$ in fünf gleiche Teile eingeteilt, so sind diese Teile klei- 
ner als wenn die Strecke in drei gleiche Teile eingeteilt wird. Demzufolge ist $\frac{1}{5}$ kleiner als $\frac{1}{3}$, was bei gleicher Anzahl der Teile bedeutet, dass $\frac{2}{5}$ kleiner als $\frac{2}{3}$ ist. Vergleich über einen Zwischenwert: Brüche können über den Vergleich eines Zwischenwertes als Bezugsgröße miteinander verglichen werden. Zum Beispiel ist $\frac{2}{5}$ kleiner als $\frac{2}{3}$, da $\frac{2}{5}$ kleiner ist als der Zwischenwert $\frac{1}{2}$ und $\frac{2}{3}$ größer ist als $\frac{1}{2}$. Somit ist $\frac{2}{5}$ kleiner als $\frac{2}{3}$.

Vergleich über den Abstand zu einem gemeinsamen Referenzwert: Brüche können über den Vergleich ihres Abstands zu einem gemeinsamen Bezugswert, wie zum Beispiel 1 miteinander verglichen werden: Beim Vergleich der Brüche $\frac{4}{5}$ und $\frac{2}{3}$ kann so etwa festgestellt werden, dass jeweils noch ein Teil bis zu einem Ganzen bzw. zu 1 fehlt. Ähnlich wie beim Vergleich von Brüchen mit einem gemeinsamen Zähler reicht es nun aus, die Stammbrüche $\frac{1}{5}$ und $\frac{1}{3}$ miteinander zu vergleichen. Da $\frac{1}{5}$ kleiner als $\frac{1}{3}$ ist fehlt beim Bruch $\frac{4}{5}$ entsprechend weniger zu einem Ganzen bzw. zu 1 als beim Bruch $\frac{2}{3}$ und folglich ist $\frac{2}{3}$ kleiner als $\frac{4}{5}$.

Padberg und Wartha (2017, S. 61 f.) sowie auch Clarke und Roche (2009) weisen darauf hin, dass die Entwicklung anschauungsbezogener und flexibler Strategien zum Größenvergleich von Brüchen reichhaltige Möglichkeiten zur Vertiefung von Bruchzahlkonzepten bietet. Aus diesem Grund sollte in der unterrichtlichen Einführung des systematischen Größenvergleichs der Schwerpunkt nicht auf dem schematischen Verfahren der Hauptnennerbestimmung mit anschließendem Vergleich der Zähler, sondern auf dem Auffinden kreativer und individueller Zugänge und Strategien liegen. Clarke und Roche (2009, S. 135 f.) fordern entsprechend mit Bezug auf Moss und Case (1999, S. 142):

„A greater emphasis on the meaning or semantics of rational numbers than on procedures for manipulating them; [... and] a greater emphasis on children's natural ways of viewing problems and their spontaneous solution strategies“.

Insgesamt ist festzuhalten, dass der Größenvergleich und das Ordnen von Brüchen eine tragfähige und flexible Vorstellungsgrundlage von Brüchen und Bruchzahlen erfordert. In Anlehnung an Postel et al. (1985, S. 19) können folgende Anforderungen an das Denken der Lernenden zusammengefasst werden:

(1) Gedankliche Flexibilität beim Übersetzen zwischen verschiedenen Repräsentationsformen von Brüchen, d.h. zwischen symbolischen Zahldarstellungen und ikonischen Veranschaulichungen, 
(2) gedankliche Flexibilität bei Umwandlungen innerhalb einer Darstellung (z. B. Erweitern von Brüchen auf symbolischer Ebene und Verfeinern der Einteilung einer ikonischen Bruchdarstellung) und

(3) zunehmende Loslösung von Anschauungsobjekten.

Die Komplexität der Anforderungen, die das Ordnen und Vergleichen von Brüchen und Bruchzahlen für die Lernenden darstellt, ist ein wesentlicher Grund dafür, dass die Fähigkeiten der Lernenden in diesem Bereich in einer Vielzahl empirischer Untersuchungen als Indikator für die Ausprägung der Entwicklung von Bruchzahlkonzepten und einem verständigem Umgang mit Brüchen genutzt werden (vgl. Obersteiner \& Tumpek, 2016; Van Hoof, Verschaffel \& Van Dooren, 2015; Clarke \& Roche, 2009; Cramer, Post \& delMas, 2002).

\subsubsection{Schwierigkeiten in der Entwicklung des Bruchzahlbegriffs}

Die Einführung von Brüchen bzw. die Zahlbereichserweiterung von $\mathbb{N}$ nach $\mathbb{Q}^{+}$ist ein schwieriger Prozess, der konzeptuelle Erweiterungen von elementaren Zahl- und Operationsvorstellungen erfordert. Es müssen sukzessive neue Grundvorstellungen aufgebaut werden, die im Verlauf der fortschreitenden Begriffsbildung erweitert, miteinander vernetzt, neu strukturiert und zum Teil korrigiert werden müssen. Aus diesem Grund ist die Bruchrechnung wohl eines der meist untersuchten Inhaltsgebiete in der Mathematikdidaktik und viele Fehlermuster und Fehlkonzepte sind bekannt. Im Folgenden werden die wesentlichen Problembereiche und Schwierigkeiten in der Entwicklung des Bruchzahlbegriffs vor dem Hintergrund des Aufbaus von Grundvorstellungen erörtert.

\section{Grundvorstellungsanpassungen von $\mathbb{N}$ nach $\mathbb{Q}^{+}$}

Die Zahlbereichserweiterung von den natürlichen Zahlen zu den positiven rationalen Zahlen ist geprägt von den Unterschieden zwischen diesen Zahlbereichen. In Hinsicht auf eine erfolgreiche Begriffsentwicklung und den flexiblen Umgang mit Brüchen ist es wichtig diese zu identifizieren und zu thematisieren (Prediger, 2004). Auf der Ebene von Grundvorstellungen bzw. „Grundüberzeugungen“ (Winter, 1999) ist es notwendig die strukturellen Änderungen der Zahlen und Rechenoperationen auf die Ebene der mentalen Strukturen zu überführen und bestehende Grundvorstellungen an den neuen Zahlbereich anzupassen und zu verändern. Wartha (2007, S. 237) spricht in diesem Zusammenhang von „Grundvorstellungsumbrüchen“. Das Ausbleiben notwendiger Grundvorstellungsanpassungen kann die Entwicklung von 
stabilen Fehlvorstellungen zur Folge haben, die den Umgang und das Operieren mit Bruchzahlen ,bewusst und unbewusst beeinflussen“ (Wartha \& Güse, 2009, S. 265).

Die zentralen Unterschiede zwischen natürlichen Zahlen und Bruchzahlen betreffen vor allem folgende Eigenschaften (vgl. Winter, 1999; Stafyladou \& Vosniadou, 2004; Prediger, 2004; Wartha, 2007; Prediger, 2008; Wartha \& Güse, 2009) ${ }^{9}$ :

Notation: In den natürlichen Zahlen ist die Schreibweise für eine Zahl eindeutig. Jede Zahl hat genau ein Zahlzeichen, das sich eindeutig aus einer Folge von Stellenwertangaben ergibt, z. B. $72=70+2$. Bruchzahlen hingegen lassen sich durch unendlich viele Repräsentanten darstellen, z. B. $\frac{1}{2}=\frac{2}{4}=\frac{3}{6}=\frac{1 \cdot m}{2 \cdot m}$ mit $m \in \mathbb{N}$. Zudem erhalten auch natürliche Zahlen durch die Bruchschreibweise eine unendliche Anzahl neuer Bezeichnungen.

Zahlaspekte: Natürliche Zahlen beantworten in der Regel die Frage „Wie viele?“ (Kardinalzahlaspekt) oder „Der wie vielte?“ (Ordinalzahlaspekt). Im Gegensatz dazu können Brüche unter verschiedenen Zahlaspekten betrachtet werden, z. B. Anteil, Operator, Verhältnis, etc. (siehe oben).

Ordnung und Dichte: Jede natürliche Zahl (außer 1) hat einen eindeutig bestimmten Vorgänger und Nachfolger. Hingegen lassen sich zwischen zwei beliebigen Bruchzahlen unendlich viele Bruchzahlen finden, die zwischen den beiden liegen. Daher kann kein direkter Vorgänger und Nachfolger bestimmt werden. Aus diesem Grund ist der Größenvergleich von natürlichen Zahlen über kardinale und ordinale Überlegungen direkt möglich, während er bei Bruchzahlen nur indirekt über gemeinsame Nenner (bzw. Unterteilungen) oder dem Vergleich mit Bezugszahlen (oder Zwischenzahlen) möglich ist.

Die unreflektierte Übertragung von Struktureigenschaften der natürlichen Zahlen auf die Bruchzahlen ist ein wesentlicher Auslöser für die Entwicklung von Fehlvorstellungen und deren Verfestigung (Wartha, 2007, S. 237). In experimentellen Studien wird die Übertragung von Strukturelementen von den natürlichen auf die rationalen Zahlen auch als ,natural number bias“ bezeichnet (z. B. Van Hoof, Verschaffel \& Van Dooren, 2015; Van Hoof, Vanderwalle, Verschaffel \& Van Dooren, 2015; Obersteiner, Van Dooren, Van Hoof \& Verschaffel, 2013; Vamvakoussi, Van Dooren \& Verschaffel, 2012).

\footnotetext{
${ }^{9}$ Die hier aufgeführten Unterschiede beziehen sich vornehmlich auf die Eigenschaften von Zahlen. In Hinsicht auf das Operieren mit Zahlen lassen sich weitere grundlegende Unterschiede beschreiben, die jedoch nicht Gegenstand dieser Arbeit sind.
} 


\title{
Fehlvorstellungen und typische Fehlerstrategien
}

Zahlreiche empirische Arbeiten beschreiben fehlerhafte Denkmuster beim Verständnis und Fehlerstrategien bei der Anwendung des Bruchzahlbegriffs ${ }^{10}$. In vielen Fällen liegt die Ursache für diese Fehler in Fehlvorstellungen, die während der Entwicklung des Bruchzahlbegriffs und der Ausbildung von Grundvorstellungen durch das Ausbleiben von Grundvorstellungsanpassungen entstehen und sich im weiteren Lern- und Übungsverlauf verfestigen (Wartha, 2007, S. 237). Im Folgenden werden ausgewählte Fehlvorstellungen und Fehlerstrategien zu Bruchzahlen und zum Umgang mit ihnen dargestellt.

Darstellen und Ablesen von Brüchen: Bei der sogenannten Teil-zu-Teil-Strategie wird die Anzahl der markierten Teile in das Verhältnis zu den nicht-markierten Teilen einer Darstellung gesetzt (Eichelmann, Narciss, Schnaubert \& Melis, 2012, S. 40 f.). Entsprechend wird der Bruch $\frac{2}{5}$ nicht als Anteil, sondern als Verhältnis (2 : 3) interpretiert: , $\frac{2}{3}$ bedeutet 2 gefärbte und 3 ungefärbte Stücke“ (Wartha, 2007, S. 52). Die Fehlvorstellung steht in engem Bezug zu der Fehlvorstellung $\frac{1}{n}$ bezeichnet eine Menge von $n$ Objekten (Wartha, 2007, S. 53; vgl. auch Hasemann, 1997, S. 13; Eichelmann, Narciss, Schnaubert \& Melis, 2012, S. 40 f.). Hierbei wird zum Beispiel der Stammbruch $\frac{1}{4}$ als vier Teile übersetzt. Eine weitere Ausprägung dieser Fehlvorstellung ist, dass in Darstellungen von echten Brüchen die Anzahl der markierten Teile ohne Bezug zur Gesamtzahl der Teile angegeben wird. Sind zum Beispiel von sieben Teilen vier Teile markiert, wird diese Darstellung nicht als Bruch $\frac{4}{7}$, sondern als 4 übersetzt (Eichelmann, Narciss, Schnaubert \& Melis, 2012, S. 40 f.). Die Ursache für diese beiden Fehlvorstellungen wird in einem Bezug auf die Abzählbarkeit der natürlichen Zahlen beschrieben:

\begin{abstract}
„Diese Strategie ist bei schwächeren Schülerinnen und Schülern weit verbreitet und darauf zurückzuführen, dass sie in Ermangelung eines adäquaten Bruchzahlbegriffs der Bruchrechnung durch Rückgriff auf abzählbare Mengen, also auf ihre Kenntnisse über natürliche Zahlen auszuweichen versuchen.“ (Hasemann, 1997, S. 13)
\end{abstract}

Besonders zu Beginn der Begriffsbildung und dem Aufbau der Anteilvorstellung anhand von ikonischen Flächenrepräsentationen ist zu beobachten, dass Lernende lediglich die Anzahl der Teilflächen betrachten, ohne die Aufteilung der Fläche zu berücksichtigen (Herden \& Pallack, 2000, S. 265; Eichelmann, Narciss, Schnaubert \& Melis, 2012, S.41). Die Nichtberücksichtung der gleichmäßigen Einteilung des Ganzen ist auf die unzureichende Ausprägung der Anteilvorstellung zurückzuführen

${ }^{10}$ Eine ausführliche Übersicht findet sich bei Eichelmann et al. (2012). 
und deutet darauf hin, dass die Lernenden noch nicht erfasst haben, dass die Größe der Teile von Bedeutung für die Angabe eines Anteils vom Ganzen ist.

Die Fehlvorstellung $\frac{m}{n}$ bedeutet $m$ Ganze, die in $n$ Teile geteilt sind ist auf die fehlerhafte Interpretation der Bedeutungen von Zähler und Nenner zurückzuführen (Wartha, 2007, S. 53; vgl. auch Mack, 1995):

„Der Zähler des Bruchs wird hierbei nicht als Anzahl der betrachteten Stücke interpretiert, sondern als Beschreibung der Anzahl der Grundmenge. Demnach ist $\frac{1}{3}$ eine Schreibweise für ,eine Pizza, die in drei Stücke geteilt ist“ oder $\frac{3}{8}$ „,drei Kürbisse, die in acht Stücke geschnitten sind““" (Wartha, 2007, S. 53)

Operatorvorstellung: Die größte Schwierigkeit bei der operativen Anteilbildung sieht Wartha (2007, S.57f.) in der Wahl der falschen Rechenoperation: „Es ist nicht selbstverständlich, dass das „Anwenden eines Operators“ und „Multiplizieren" einen synonymen Zusammenhang suggeriert, da auch andere Operationen von Zahlen auf Größen denkbar sind“" (Wartha, 2007, S. 57 mit Verweis auf Seyfferth, 1975). Da die Anwendung von Bruchoperatoren in vielen Fällen anhand von Brüchen zwischen 0 und 1 eingeführt wird, die eine Verkleinerung der Ausgangsgröße bewirken, werden mit der Anteilbildung vor allem die Division und die Subtraktion verbunden: ,Zur Anteilbestimmung werden Rechenoperationen ausgewählt, die bei natürlichen Zahlen verkleinernd wirken" (Wartha, 2007, S. 232). Die Ursache dafür wird ,in der Verhaftung der Grundvorstellung der Multiplikation als wiederholtes Reproduzieren von Gegenständen“ (Wartha, 2007, S. 57) beschrieben, die auf das Operieren mit natürlichen Zahlen zurückgeführt werden kann und eine Vermehrung der Ausgangsgröße bewirkt. Demgegenüber erfordert die Interpretation eines Bruchs als Operator die Vorstellung von der Multiplikation als ,,kontinuierliche Veränderung" im Sinne einer Streckung und Stauchung (vom Hofe, 1995, S. $120 \mathrm{ff}$;; vgl. auch Griesel, 1973). Diese Vorstellung birgt jedoch Schwierigkeiten bei der Anwendung auf diskrete Größen: So bedeutet $\frac{3}{4}$ von 12 Stücken Kuchen nicht, dass 12 Kuchenstücke auf 12 Kuchenstücke abgebildet werden, deren Größe $\frac{3}{4}$ der Ausgangsgröße ist, sondern, dass 12 Kuchenstücke auf 9 Kuchenstücke derselben Größe abgebildet werden (vgl. Wartha, 2007, S. 58 f.).

Eine weitere Schwierigkeit bei der operativen Anteilbildung beschreiben Behr et al. (1993) in der komponentenweisen Betrachtung von Brüchen als Kombination von zwei Zahlen:

„We suspect that one of the difficulties students have in understanding rational number is due to the fact that the symbolic representation of a rational number involves two numbers. Yet a rational number represents a single quantity, or in the case of rational 
number as operator, a single function. It has been hypothesized that many errors that children make with rational number operations and relations is due to the fact that they perceive a rational number as two entities.“ (Behr, Post, Harel \& Lesh, 1993, S. 46)

Die komponentenweise Betrachtung von Brüchen als Kombination zweier (natürlicher) Zahlen bedeutet, dass der Zähler und der Nenner eines Bruchs nicht als Teiloperatoren betrachtet werden, die verkettet auf eine Größe wirken. Dies führt in vielen Fällen zur Übertragung von Struktureigenschaften aus den natürlichen Zahlen, die wiederum zur Wahl der falschen Rechenoperation führen (siehe oben).

Erweitern und Kürzen: In vielen Fällen gelingt es Lernenden nicht, inhaltliche Vorstellungen für das Erweitern und Kürzen von Brüchen als Verfeinern und Vergröbern einer Einteilung auszubilden. Im Gegensatz dazu bereitet ihnen die Durchführung des technischen Verfahrens auf symbolischer Ebene zumeist keine Schwierigkeiten. Padberg (2009, S. 56) berichtet Lösungsquoten von über $90 \%$ in Aufgaben, in denen das Erweitern und Kürzen von Brüchen auf symbolischer Ebene isoliert abgefragt wird. Die technische Beherrschung des Verfahrens erlaubt dabei jedoch keinen Rückschluss auf inhaltliche Vorstellungen, die mit dem Erweitern und Kürzen von Brüchen auf Zahlenebene verbunden werden, sowie auf das inhaltliche Verständnis von äquivalenten Brüchen (vgl. Wartha, 2007, S. 71; Eichelmann, Narciss, Schnaubert \& Melis, 2012, S. 44).

Bezogen auf das inhaltliche Verständnis für das Erweitern und Kürzen von Brüchen beschreibt Wartha (2007, S. 69f.) die zentrale Fehlvorstellung durch Erweitern und Kürzen verändert sich der Wert des Bruchs. Wartha (2007, S. 69) bezieht sich in seiner Beschreibung dieser Fehlvorstellung auf eine repräsentative Untersuchung von Payne (1986, S.53), in der über 70\% der untersuchten 13-Jährigen und über die Hälfte der untersuchten 17-Jährigen Schülerinnen und Schüler angaben, dass das Verdoppeln von $a$ und $b$ im Bruch $\frac{a}{b}$ dazu führe, dass sich auch der Wert des Bruchs verdoppele. Padberg und Wartha (2017) weisen darauf hin, dass diese Fehlvorstellung womöglich darauf zurückzuführen ist, dass die Begriffe ,Erweitern“ und „Kürzen“ im alltäglichen Sprachgebrauch stets mit einer Wertänderung verbunden werden. Wenn zum Beispiel ein Grundstück erweitert wird, so vergrößert sich die Grundstücksfläche und analog bekommt ein Arbeitnehmer weniger Gehalt, wenn dieses zuvor gekürzt wurde (vgl. Padberg \& Wartha, 2017, S.52; Wartha, 2007, S. $69 \mathrm{f}$.).

In einer Interviewstudie berichtet Hart (1987, S.6) zudem, dass bereits drei Monate, nachdem die Lernenden das Erweitern von Brüchen durch Multiplikation von Zähler und Nenner mit dem gleichen Faktor erarbeitet hatten, keiner der untersuchten Lernenden diese Methode zur Angabe von zu $\frac{1}{3}$ äquivalenten Brüchen 
angewendet hat. Stattdessen zeigten die Lernenden additive Strategien zum Erweitern von Brüchen sowie individuell entwickelte und oftmals fehlerhafte Verfahren (Hart, 1987, S. 6; vgl. auch Eichelmann et al., 2012, S. 45).

Bezogen auf das inhaltliche Verständnis für die Wertgleichheit bzw. Äquivalenz von Brüchen führt Wartha (2007, S. 70) die Fehlvorstellung Zwei Brüche sind äquivalent, wenn die Differenzen von Zähler und Nenner gleich sind an. Aus ihren Interviewstudien berichtet Hart (1987, S.6) unter anderem, dass die Brüche $\frac{6}{16}$ und $\frac{9}{19}$ als äquivalent bewertet wurden, da die Differenzen der Zähler und Nenner jeweils 10 betragen und somit gleich seien. Insgesamt bezeichneten in dieser Studie nur fünf von zwölf Lernenden drei Monate nach der unterrichtlichen Behandlung des Erweiterns und Kürzens von Brüchen den Bruch den $\frac{10}{14}$ als äquivalent zum Bruch $\frac{5}{7}$, während drei Lernende angaben, dass $\frac{10}{14}$ sowohl äquivalent als auch das Doppelte von $\frac{5}{7}$ sein könne. Auch diese Fehlvorstellung kann auf die Anwendung von Additions- und Zählstrategien zurückgeführt werden. Die Brüche werden als Kombinationen natürlicher Zahlen und nicht als eigene Zahlen wahrgenommen und Zusammenhänge zwischen den natürlichen Zahlen in Zähler und Nenner gesucht. Payne (1986) weist in diesem Zusammenhang darauf hin, dass eine Ursache in der Notation von Folgen äquivalenter Brüche liegen könnte (wie z. B. $\frac{1}{2}, \frac{2}{4}, \frac{3}{6}, \frac{4}{8}, \ldots$ ). Diese würden eher einen additiven als einen multiplikativen Zusammenhang suggerieren.

Weitere häufig festgestellte Fehlertypen beim Erweitern und Kürzen von Brüchen können auf Assoziationsfehler (Schaffrath, 1961, S. 16 ff.) zurückgeführt werden:

„Jede Vorstellungsreproduktion stützt sich auf die konservierende Fähigkeit des Bewußtseins, die man als Gedächtnis bezeichnet. Das Gedächtnis wiederum hängt stark von den Verbindungen seiner Inhalte untereinander ab, den sogenannten Assoziationen. Solche falschen Assoziationen können zustande kommen, wenn von einem Zahlwort mehrere verschiedenartige Tendenzen zur Reproduktion ausgehen, indem sie sich gegenseitig stören [...].“ (Schaffrath, 1961, S. 16)

Assoziationsfehler äußern sich u.a. im Erweitern zur Identität, wie im Beispiel $\frac{4}{7}+\frac{1}{4}=\frac{16+28}{28}=\frac{44}{28}$ (Eichelmann, Narciss, Schnaubert \& Melis, 2012, S. 45), in dem der Bruch $\frac{1}{4}$ nicht zu $\frac{7}{28}$, sondern zur Identität $\frac{28}{28}$ erweitert wird. Weitere Beispiele finden sich bei Padberg und Wartha (2017, S. 53), die von einer Untersuchung berichten, in der rund ein Fünftel aller Lernenden den Bruch $\frac{9}{18}$ fehlerhaft zu $\frac{1}{9}$ oder $\frac{9}{18}=\frac{3}{6}=\frac{1}{3}$ kürzten. Im Ersten Fall ist die Zahl 9 beim Kürzen so dominant, dass die Lernenden lediglich den Zähler richtig kürzen und den Faktor 9 in den Nenner schreiben. Im zweiten Fall ist Kürzungsfaktor 3 so dominant, dass der Bruch $\frac{3}{6}$ nicht 
zu $\frac{1}{2}$, sondern zu $\frac{1}{3}$ gekürzt wird (Padberg \& Wartha, 2017, S. 70; weitere Beispiele beschreiben Eichelmann, Narciss, Schnaubert \& Melis, 2012, S. 45 f.).

Die hier beschriebenen Fehlvorstellungen zum Kürzen und Erweitern treten in empirischen Untersuchungen selten in Isolation auf, sondern sind zumeist in Aufgaben zur Addition und Subtraktion sowie insbesondere beim Größenvergleich von Brüchen zu beobachten.

Vergleichen und Ordnen: Brüche werden zumeist über die Bildung eines Hauptnenners miteinander verglichen. Dieses Verfahren ist jedoch sehr abhängig von auswendig gelernten Regeln, deren Anwendung zumeist kein Verständnis von Brüchen erfordert, da sich die notwendigen Operationen auf das Rechnen mit natürlichen Zahlen beschränken (vgl. Wartha, 2007, S. 75). Die Hauptursache für Fehler und Fehlerstrategien liegen in vielen Fällen in der Übertragung von Struktureigenschaften der natürlich Zahlen (,natural number bias“, siehe oben):

„[...] learners are found to make systematic mistakes specifically in rational number tasks where reasoning purely in terms of natural numbers results in an incorrect solution. At the same time, much higher accuracy levels are found in rational number tasks where reasoning merely in terms of natural numbers results in a correct solution." (Van Hoof, Verschaffel \& Van Dooren, 2015, S. 40)

Die Befunde zur Übertragung von Eigenschaften der natürlichen Zahlen auf Bruchzahlen, insbesondere beim Größenvergleich und Ordnen von Brüchen, weisen in vielen Fällen auf eine getrennte Betrachtung von Zähler und Nenner als voneinander unabhängige Zahlen hin. Vor diesem Hintergrund beschreibt Wartha (2007, S. 76 f.) vier häufig vorkommende Fehlvorstellungen (vgl. auch Padberg \& Wartha, 2017, S. 65 f.; Eichelmann, Narciss, Schnaubert \& Melis, 2012, S. 42 ff.):

Je kleiner der Nenner, desto größer der Bruch: Bei dieser Fehlvorstellung werden Größenvergleiche allein auf Grundlage des Nenners als Maß für die Größe der Teile und ohne Berücksichtigung des Zählers als Anzahl der betrachteten Teile vorgenommen. Obgleich diese Vorstellung beim Vergleich von Brüchen mit gleichen Zählern, wie zum Beispiel $\frac{3}{8}<\frac{3}{5}$, zielführend ist, so kann sie nicht auf den Vergleich von Brüchen mit unterschiedlichen Zählern und Nennern, wie z. B: $\frac{5}{8}$ und $\frac{2}{5}$ verallgemeinert werden. Diese Fehlvorstellung kann zum einen als Übergeneralisierung des Größenvergleichs von Stammbrüchen aufgefasst werden. Zum anderen kann eine Ursache für die alleinige Orientierung am Nenner, respektive der Größe der Teile, auf anschauliche Überlegungen zurückgehen, bei denen nur die Größe und nicht die Anzahl der betrachteten Teile eines Ganzen 
oder einer Größe verglichen werden. Diese Fehlvorstellung gilt als häufigste Fehlerstrategie beim Vergleich von Brüchen. Repräsentativ für diese Beobachtung ist ein Item aus der PALMA-Studie, bei dem die Lernenden die größte unter den Zahlen $\frac{4}{5}, \frac{3}{4}, \frac{5}{8}$ und $\frac{7}{10}$ ankreuzen sollten. Nur ein Drittel der ca. 2400 Schülerinnen und Schüler der siebten Jahrgangsstufe konnte diese Aufgabe richtig lösen und die häufigste Antwort über alle Schulformen hinweg war $\frac{3}{4}$, da dieser Bruch den kleinsten Nenner in der vorgegebenen Menge von Zahlen hat (Wartha, 2007, S. $173 \mathrm{ff}$.).

Je größer der Nenner, desto größer der Bruch: Diese Fehlvorstellung kann durch die fehlerhafte Übertragung der Ordnung der natürlichen Zahlen auf Bruchzahlen erklärt werden: $\frac{1}{2}<\frac{1}{3}$, weil 2 der Vorgänger von 3 ist. Analog zur zuvor geschilderten Fehlvorstellung wird insbesondere beim Vergleich von Brüchen, die keine Stammbrüche sind, der Zähler nicht in den Größenvergleich einbezogen. Hartnett und Gelman (1998, S. 370) berichten zudem von einer Dominanz von Zählstrategien, die zur Folge haben, dass z.B. aufgrund einer irrtümlich angenommenen Vorgänger-Nachfolger-Beziehung angegeben wird, dass $\frac{2}{2}<\frac{3}{4}$ ist, da 2 und 2 weniger sind als 3 und 4.

Je größer der Zähler, desto größer der Bruch: Bei dieser Fehlerstrategie wird der Nenner beim Vergleich von Brüchen nicht berücksichtigt und ein Größenvergleich allein durch Vergleich der Zähler nach den Ordnungsrelationen der natürlichen Zahlen vorgenommen. Entsprechend wird der Blick einseitig auf die Anzahl der Teile gerichtet, ohne die Größe der Teile einzubeziehen. Vor diesem Hintergrund wird zum Beispiel angenommen, dass, , $\frac{3}{5}<\frac{6}{10}$ “ ist, da 6 Teile mehr als 3 Teile sind (Post et al., 1985, S. 33), oder dass " $\frac{3}{12}=\frac{3}{4}$ “ ist, da in beiden Brüchen die gleiche Anzahl von Teilen betrachtet wird (Post et al., 1985, S.29).

Größere Zahlen in Zähler und Nenner bedeuten einen größeren Bruch: Auch bei dieser Fehlerstrategie werden die Zähler und Nenner der zu vergleichenden Brüche getrennt voneinander betrachtet: „Bei gleich lautender Ordnung im Zähler und Nenner wird diese Ordnung auf die Brüche übertragen (Beispiel $\frac{3}{5}<\frac{5}{12}$, da $3<5$ und $5<12$ gilt)“ (Padberg \& Wartha, 2017, S. 65). In einem Item der PALMA-Studie, in der die Lernenden aus der Menge $\frac{4}{5}, \frac{3}{4}, \frac{5}{8}$ und $\frac{7}{10}$ den größten Bruch ankreuzen sollten, wählten im Durchschnitt 11,5\% den Bruch $\frac{7}{10}$ als größten Bruch, da dieser sowohl im Zähler wie auch im Nenner die größten Zahlenwerte aufweist (Wartha, 2007, S. 175). Ähnliche Ergebnisse berichtet auch Payne (1986). In einer ähnlichen Testaufgabe, in der die Lernenden die größte Bruchzahl aus der Auswahl $\frac{2}{3}, \frac{3}{4}, \frac{4}{5}$ und $\frac{5}{8}$ angeben sollten, war der Hauptfehler in den Antworten der 9-Jährigen die Angabe des Bruchs $\frac{5}{8}$. Die Autoren berichten zudem, dass die Häufigkeit dieses Fehlertyps nach der systematischen Behandlung der Bruchrechnung im Unterricht deutlich abgenommen hat. 
Neben den hier beschriebenen Fehlvorstellungen sind in der Literatur zum GröBenvergleich und Ordnen von Brüchen noch weitere Fehlermuster dokumentiert. Ein Fehler, der auf die im Zusammenhang mit der Äquivalenz von Brüchen aufgeführten Fehlvorstellung „Zwei Brüche sind äquivalent, wenn die Differenzen von Zähler und Nenner gleich sind“" im Zusammenhang steht, wird von Padberg und Wartha (2017, S. 66) als Ergänzung zum Ganzen beschrieben. Mit Bezug auf eine Studie von Mack (1993) führen sie ein Beispiel an, in dem die Schülerinnen und Schüler die Brüche $\frac{4}{5}$ und $\frac{5}{6}$ als wertgleich betrachten, da beiden Brüchen jeweils ein Teil zum Ganzen fehlt. Hierbei werden lediglich die Anzahlen der zum Ganzen fehlenden Teile betrachtet, ohne Bezug zur Größe der jeweiligen Teile zu nehmen. Eine andere Begründung führen Padberg und Krüger (1997) an, die in ihrer Untersuchung zu denselben Brüchen die Begründung dokumentiert haben, dass die Brüche gleich groß sind, da der Unterschied zwischen Zähler und Nenner jeweils gleich groß sei, nämlich 1.

Der häufigste Fehler in der oben geschilderten PALMA-Aufgabe zu Auswahl des größten Bruchs aus der Menge $\frac{4}{5}, \frac{3}{4}, \frac{5}{8}$ und $\frac{7}{10}$ war die Angabe des Bruchs $\frac{3}{4}$, den im Durchschnitt 47,2\% der befragten Schülerinnen und Schüler wählten. Wartha (2007, S. 176) führt als Begründung für diesen Fehler die Orientierung an Brüchen aus dem Alltag an. Er vermutet,

„dass [...] viele Schüler über ein eingeschränktes Repertoire an Brüchen verfügen $\left(\frac{1}{2}, \frac{1}{4}\right.$ und $\frac{3}{4}$, eventuell noch $\frac{1}{3}$ und $\left.\frac{2}{3}\right)$, die für sie mit Bedeutung gefüllt sind und [für die] auch eine Größenvorstellung vorliegt. Andere Brüche werden nur als zusammenhangslos kombinierte Symbole wahrgenommen und sind nicht mit Grundvorstellungen verbunden.“ (Wartha, 2007, S. 176)

Aus diesem Grund nimmt Wartha an, dass der Bruch $\frac{3}{4}$ die größte Bruchzahl in der Auswahl war, die von den Lernenden inhaltlich interpretiert werden konnte, und deshalb als größte der vorgegebenen Bruchzahlen gewählt wurde.

Eine wesentliche Verständnishürde für die Erfassung der Ordnung von Bruchzahlen ist die veränderte Dichte von Zahlen bei der Zahlbereichserweiterung von den natürlichen Zahlen zu den Bruchzahlen. Während sich die natürlichen Zahlen durch die Eigenschaft auszeichnen, dass jede Zahl mit Ausnahme der Null einen eindeutig bestimmten Vorgänger und Nachfolger hat, gilt diese Vorgänger-NachfolgerBeziehung für Bruchzahlen nicht mehr, sondern zwischen zwei Bruchzahlen gibt es unendlich viele Bruchzahlen. Als Folge einer ausbleibenden Grundvorstellungsanpassung bezüglich der Dichte von Bruchzahlen beschreibt Wartha (2007, S. $80 \mathrm{f}$.) die Fehlvorstellung zwischen Brüchen wie $\frac{1}{3}$ und $\frac{1}{2}$ oder $\frac{3}{5}$ und $\frac{4}{5}$ kann kein Bruch angegeben werden - Diese Brüche werden als Nachbarzahlen verstanden. Auch 
diese Fehlvorstellung kann mit einer getrennten bzw. isolierten Betrachtung der Zählers und des Nenners erklärt werden. Diese werden nicht in Bezug zueinander gesetzt, sondern als unabhängige natürliche Zahlen betrachtet, weshalb die Vorgänger-Nachfolger-Beziehung der natürlichen Zahlen auf die Bruchzahlen übertragen wird:

„Die Mehrzahl der Schüler verharrt in den Vorstellungen zu natürlichen Zahlen, die grundlegende Revision des Vorwissens (jede natürliche Zahl hat einen Vorgänger und Nachfolger) für die Bruchzahlen (zwischen zwei Brüchen ist immer ein weiterer Bruch) fand nicht statt.“ (Wartha, 2007, S. 81)

Insgesamt wird deutlich, dass die empirisch festgestellten und beschriebenen Hauptschwierigkeiten beim Größenvergleich und Ordnen von Brüchen in einer getrennten Betrachtung von Zähler und Nenner als natürliche Zahlen bestehen. Brüche werden nicht als eigene Zahlen behandelt, sondern als Kombinationen von natürlichen Zahlen interpretiert. Dies führt zu der fehlerhaften Übertragung von Struktureigenschaften der natürlichen Zahlen auf die Bruchzahlen. Notwendige Grundvorstellungsanpassungen bleiben aus.

\subsubsection{Zusammenfassung}

Die sachanalytischen Überlegungen und die Diskussion der empirischen Befunde zur Entwicklung des Bruchzahlbegriffs im vorhergehenden Abschnitt lässt sich in der Ableitung von drei Folgerungen für die Erarbeitung des Bruchzahlbegriffs zusammenfassen:

- Der Aufbau von Grundvorstellungen zu Bruchzahlen erfordert die Ausbildung und Stärkung von Bezügen zu Anwendungskontexten und der Erfahrungswelt der Lernenden (Sinnstiftung).

- Durch die Verknüpfung von verschiedenen Darstellungen und Repräsentationsebenen werden mentale Handlungsmodelle zu Bruchzahlen und zentralen Operationen mit Brüchen aufgebaut (Mentale Repräsentation).

- Um der Entwicklung von Fehlvorstellungen vorzubeugen müssen bestehende Vorstellungen erweitert und neue Vorstellungen in das bestehende Netz von Vorstellungen integriert werden (Antizipation von Schwierigkeiten).

Der Aufbau von Grundvorstellungen zum Bruchzahlbegriff kann in vielen Fällen durch das Anknüpfen an reale Handlungen und Handlungsfolgen beschrieben wer- 
den, die Bruchzahlen und -Operationen eine Bedeutung geben. Brüche werden als Anteile eines oder mehrerer Ganzer, als Maßzahlen, Skalenwerte oder Anteile von beliebigen Größen in Anwendungszusammenhängen konzeptualisiert, denen eine gemeinsame Handlung zugrunde liegt: Dem Aufteilen des Ganzen in gleich große Teile und dem Vervielfachen eines Teils bzw. dem Auswählen einer bestimmten Anzahl von Teilen. Dabei dienen Sachsituationen einerseits als Bindeglied zur Erfahrungswelt der Lernenden. Andererseits trägt die Variation unterschiedlicher Sachkontexte zur Erweiterung der Anwendungsbezüge und auf Ebene der mentalen Strukturen der Lernenden zur Ausbildung eines allgemeinen Denkmusters bzw. zur Abstraktion und damit zur Verallgemeinerung des Herstellungsverfahrens bei.

Ziel ist es, durch die Integration von verschiedenen Sachsituationen, ikonischen und symbolischen Darstellungen mentale Repräsentationen und Handlungsmodelle aufzubauen, die es Lernenden ermöglichen auch in komplexen Situationen mental mit Bruchzahlen zu operieren und ihnen eine Bedeutung zu geben. Wesentlich hierfür ist das Übersetzen zwischen verschiedenen Darstellungen und Repräsentationsebenen. Dieses erlaubt es Lernenden unbekannte Situationen auf einen vertrauten Erfahrungsbereich zurückzuführen und sie durch ikonische Repräsentationen zu strukturieren und zu visualisieren. In Hinsicht auf eine fortschreitende Begriffsentwicklung dient das Übersetzen zwischen Darstellungen und Repräsentationsebenen der zunehmenden Abstraktion und Verallgemeinerung gemeinsamer Strukturen und soll auf diese Weise zum Aufbau mentaler Repräsentationen beitragen.

Es wurde dargestellt, dass eine Vielzahl verbreiteter Fehlermuster und Fehlvorstellungen in der Entwicklung des Bruchzahlbegriffs neben einer unzureichenden Entwicklung mentaler Modelle auf fehlerhafte Übertragungen von Struktureigenschaften der natürlichen Zahlen auf die Bruchzahlen erklärt werden können. Notwendige Grundvorstellungsanpassungen bleiben aus und neue Zusammenhänge werden auf Grundlage von vertrauten Handlungsmodellen interpretiert, die im Bereich der Bruchzahlen nicht mehr tragen. Aus diesem Grund wird empfohlen, Grundvorstellungsanpassungen und bekannte Fehlvorstellungen explizit zu thematisieren, um der Entwicklung von Fehlkonzepten vorzubeugen und die Bewältigung von erwarteten Hürden in der Begriffsbildung zu unterstützen.

\subsection{Analyse von Transferprozessen auf normativer Ebene}

$\mathrm{Zu}$ Beginn dieses Kapitels wurde das Konzept der Grundvorstellungen als ganzheitliches didaktisches Modell des Mathematiklernens dargestellt und ein Bezug zu den verschiedenen theoretischen Perspektiven zu Transfer hergestellt. Auf der Basis des Grundvorstellungsmodells wurden Transferprozesse definiert als 
Prozesse der Anwendung oder Übertragung mathematischer Begriffe, Verfahren und Strukturen aufneue Anforderungssituationen zum Transfer zwischen Sach- und Anwendungskontexten, zum Transfer zwischen Darstellungen und Repräsentationsebenen sowie zum Herstellen und Begründen von mathematischen Zusammenhängen.

In diesem Zusammenhang wurde argumentiert, dass die Anwendung und Übertragung mathematischer Begriffe, Verfahren und Strukturen die Aktivierung von Grundvorstellungen erfordern und dadurch wechselseitig zum Aufbau, zur Entwicklung und zur Verknüpfung von Grundvorstellungen beitragen. Die didaktischen Analysen und empirischen Befunde zur Entwicklung des Bruchzahlbegriffs verdeutlichen diesen Zusammenhang und stellen heraus, dass die zentralen Elemente in der Zahlbegriffsentwicklung im Übersetzen zwischen Sachkontexten und Anwendungszusammenhängen sowie im Übersetzen zwischen verschiedenen Darstellungen und Repräsentationsebenen zum Aufbau mentaler Handlungsmodelle und deren Erweiterung und Vernetzung konzeptualisiert werden können.

\section{Operative Anteilbildung:}

\section{A1:}

Julia hat 6 Achtel von ihrer Pizza gegessen. Erkläre wie viel das ist. Du kannst auch zeichnen.

\section{A2:}

Jakob hilft seinem Vater beim Kuchenbacken. Für den Kuchen benötigen sie zwei Drittel von einem Paket Butter. Zeichne ein, wie groß das benötigte Stück Butter ist.

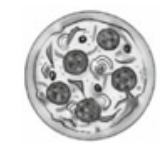

\section{A3:}

Svenja fährt mit ihrer Mutter im Zug von Bielefeld nach Hannover. Nach 50 min haben sie bereits fünf Sechstel der $120 \mathrm{~km}$ langen Strecke zurückgelegt. Wie viele $\mathrm{km}$ müssen sie noch fahren.

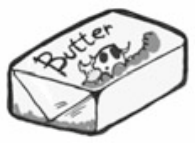

A4:

Jan und Lisa haben 10 Stunden in einem Jugendtreff gearbeitet. Jan hat vier Stunden gearbeitet und Lisa sechs Stunden. Zusammen erhalten sie $50 €$ als Lohn für ihre Arbeit. Sie wollen den Lohn gerecht nach der Anzahl der Stunden aufteilen, die sie jeweils gearbeitet haben.

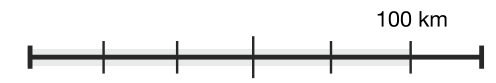

$0 \mathrm{~km}$ $120 \mathrm{~km}$ Wie viel $€$ bekommt jeder?

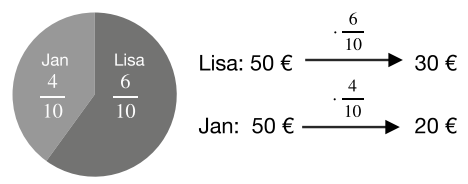

Abbildung 2.19 Aufgabenserie zur operativen Anteilbildung 
Der Sachkontext einer Aufgabe beinhaltet die situativen Eigenschaften einer Anforderung. Eine Anforderung kann einerseits innermathematisch oder als realer Sachverhalt formuliert sein. Wesentlich für den Transfer zwischen verschiedenen Sachkontexten sind die enthaltenen Größen, Darstellungen und weiteren bedeutungstragenden Elemente. Die Aufgabenbeispiele in Abbildung 2.19 sind jeweils mit Bezug zu einem alltagsbezogenen Sachkontext dargestellt, die an unterschiedliche situative Handlungskonzepte gebunden sind. In den Aufgaben 1 und 2 wird mit den Ausgangsgrößen (dem Ganzen) ein Bezug zu realen Gegenständen hergestellt: $\mathrm{Zu}$ einer Pizza, die als Kreisfläche repräsentiert werden kann und zu einem Stück Butter, das als Quader repräsentiert werden kann. Die Bildung eines Anteils ist in beiden Fällen mit der gegenständlichen Handlung des Schneidens in Teile verbunden. Demgegenüber sind die Ausgangsgrößen in den Aufgaben 3 und 4 keine gegenständlichen Repräsentationsobjekte, sondern kontinuierliche Größen: Eine Strecke mit $120 \mathrm{~km}$ Länge und ein Geldbetrag von $50 €$. Das zentrale Handlungskonzept zum Umgang mit einer Strecke ist in den meisten Fällen das Messen einer Länge und in diesem Zusammenhang das Unterteilen (Segmentieren) in Teilstücke. Zum Aufteilen eines Geldbetrags hingegen muss kein realer Handlungsprozess zugrunde liegen. Das Aufteilen in unterschiedliche Stückelungen würde den Aufteilungsprozess sogar verkomplizieren. Insofern erfordert die Darstellung und Herstellung von Anteilen in den Aufgaben 3 und 4 die Nutzung abstrakter und symbolischer Repräsentationen.

Allen Aufgaben gemein ist die zugrundeliegende Herstellungshandlung, die unabhängig davon, ob der Anteil einer Pizza oder eines Geldbetrags berechnet werden soll, dem gleichen Prinzip folgt: Ein Ganzes wird in gleiche Teile geteilt und ein Teil vervielfacht. Die Übertragung dieses Verfahrens auf die Bestimmung von Anteilen verschiedener Größen trägt zur Erweiterung der Anwendungsbezüge und auf Ebene der mentalen Strukturen zur Ausbildung eines allgemeinen Denkmusters bzw. zur Abstraktion und damit zur Verallgemeinerung des Verfahrens bei. Die Unterschiede in den Anforderungen werden durch die situativen Handlungskonzepte der betrachteten Größe als Element des Sachzusammenhangs bestimmt.

Die Aufgabenserie in Abbildung 2.19 veranschaulicht zudem, dass das Übersetzen zwischen gegenständlichen, bildlichen und symbolischen Repräsentationen mit Transferprozessen verbunden ist. Der Transfer besteht hierbei im Wesentlichen in der Loslösung des mathematischen Objekts oder eines Verfahrens aus der ursprünglichen Darstellung und der Übertragung auf eine neue Darstellung. Dies erfordert in den meisten Fällen, das Übertragene an die Eigenschaften der neuen Darstellung anzupassen.

Respektive einer zunehmenden Verallgemeinerung besteht ein weitaus schwierigerer Transferprozess im Übergang von gegenständlichen zu abstrakten und symbo- 


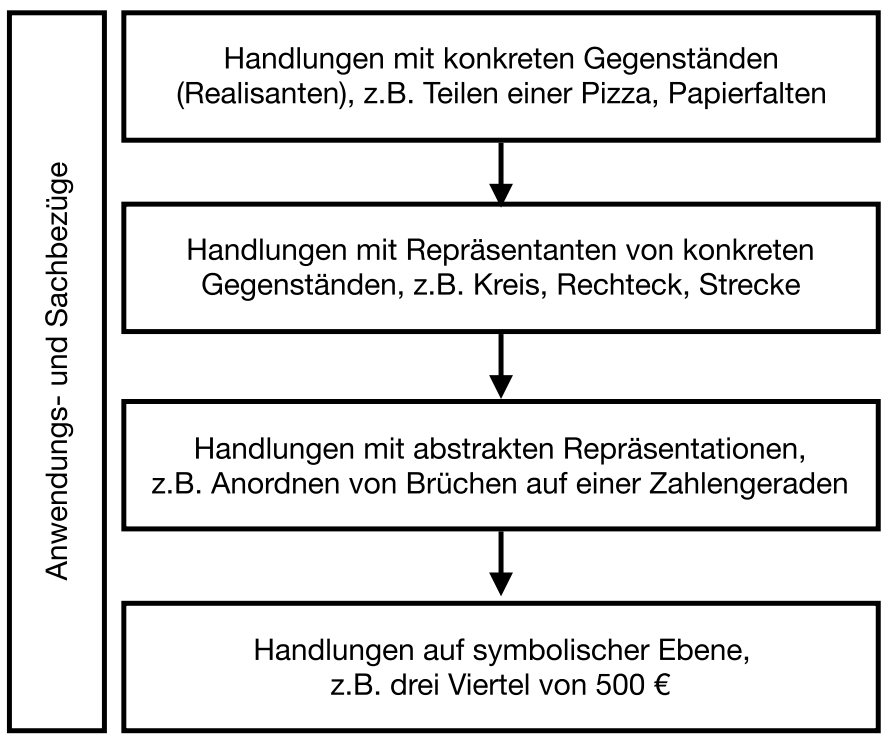

Abbildung 2.20 Allgemeine Transferschritte zur Abstraktion und zunehmenden Verallgemeinerung eines Verfahrens

lischen Repräsentationen, zum Beispiel von dem Bruchteil einer Pizza zum Anteil einer beliebigen Größe.

Vor dem Hintergrund des Aufbaus von primären Grundvorstellungen, die an gegenständlichen Handlungserfahrungen der Lernenden anknüpfen, und der Überführung in sekundäre Grundvorstellungen, die auf mathematischen Operationen mit symbolischen Objekten beruhen, ohne dabei die anschauliche Vorstellungsgrundlage aufzugeben, werden Repräsentationswechsel in der fortschreitenden Begriffsbildung zumeist über sukzessive Abstraktionen konzeptualisiert (vgl. Abb. 2.20 und Abb. 2.21).

Die Aufgabenbeispiele zur Grundvorstellung des Erweitern als Verfeinern einer Einteilung in Abbildung 2.21 veranschaulichen diese Transferschritte. Ungeachtet der ikonischen Repräsentationsform als Rechteck wird in Aufgabe 1 durch das Papierfalten ein Bezug zu einer Handlung mit realen Gegenständen hergestellt. Durch das Falten eines Blatts Papier wird die Einteilung des Papiers zunehmend verfeinert, ohne dass der gefärbte Anteil verändert wird. Das repräsentierte Verfahren des Verfeinerns der Einteilung kann durch eine Handlung mit realen Gegenständen durchgeführt oder mental simuliert werden. Im Gegensatz dazu soll in Aufgabe 


\section{Erweitern als Vergröbern einer Einteilung}

A1:

Ein Blatt Papier ist zu zwei Dritteln blau gefärbt. Welche Anteile entstehen durch Falten des Papiers in der Abbildung?
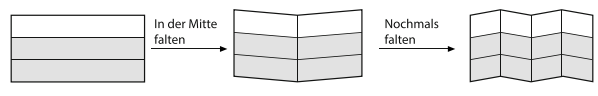

A2:

In der Abbildung siehst du drei gleich große Quadrate, die unterschiedlich eingeteilt sind. Begründe warum der schraffierte Teil der Quadrate jeweils gleich ist.
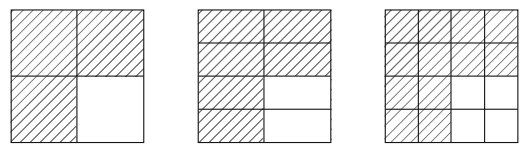

A3:

Teile die Strecke so ein, dass doppelt (dreimal, sechsmal) so viele Teile entstehen. Welcher Anteil der Strecke ist jeweils gelb unterlegt?

A4:

Erweitere die Brüche $\frac{2}{3}$ und $\frac{3}{5}$,

so dass sie den gleichen Nenner haben.

Welcher Bruch ist größer?

Du kannst auch zeichnen.

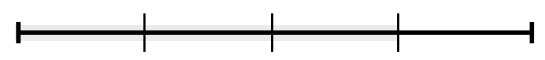

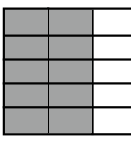

$\frac{2}{3}=\frac{10}{15}$

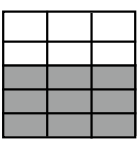

$\frac{3}{5}=\frac{9}{15}$

Abbildung 2.21 Aufgabenserie zum Erweitern von Brüchen als Verfeinern der Einteilung

2 auf Grundlage von ikonischen Darstellungen argumentiert werden, warum die schraffierten Flächen jeweils gleich sind. Durch die Notation der jeweils schraffierten Anteile kann hier bereits eine Übersetzung in die symbolische Ebene erfolgen. Aufgabe 3 stellt einen Repräsentationswechsel dar, in dem die Handlung des Verfeinerns der Einteilung eines Rechtecks auf die die ikonische Darstellung einer Strecke thematisiert wird. Während das Vorgehen im Vergleich zum Falten eines Papiers oder Einteilen eines Rechtecks im Grunde die gleiche ist, so stellt eine Strecke ein unterschiedliches Handlungskonzept dar, da die Größe keine Fläche ist, sondern die Länge einer Strecke.

In Aufgabe 4 wird das Verfahren auf die symbolische Ebene übertragen. Die Brüche sollen so erweitert werden, dass sie einen gemeinsamen Nenner haben, um festzustellen, welches die größere der beiden Bruchzahlen ist. Dieser Vergleich auf symbolischer Ebene kann durch das Übersetzen auf die ikonische Repräsentationsebene veranschaulicht werden und das Erweitern der Bruchzahlen auf einen Hauptnenner als Herstellen einer gemeinsamen Einteilung vergegenwärtigt werden. Ein wesentlicher Transferprozess hierbei besteht in der Verknüpfung der Handlung auf 
Darstellungsebene mit dem symbolischen Verfahren der Multiplikation von Zähler und Nenner mit demselben Faktor.

Die Beispiele zeigen auf, dass Transferprozesse auf normativer Ebene im Rahmen von sachanalytischen Überlegungen beschrieben werden können. Ziel dieser Überlegungen ist es, möglichst genau zu beschreiben,

- worin der konzeptuelle Kern als Gegenstand eines Transfers besteht, d. h. welche Begriffsaspekte, Verfahren oder Strukturen übertragen werden sollen,

- welche Übersetzungen auf Ebene des Sachkontexts respektive der mit ihnen verbundenen situativen Handlungskonzepte sowie zwischen Darstellungen und Repräsentationsebenen erforderlich sind und

- welche möglichen Schwierigkeiten, Übergeneralisierungen und fehlerhafte Übertragungen zu erwarten sind.

\subsection{Fazit und Forschungsdesiderata}

Das Konzept der Grundvorstellungen als mathematikdidaktisches Modell mit langer Tradition ermöglicht die Integration verschiedener psychologischer und didaktischer Modelle und Perspektiven in eine ganzheitliche Sichtweise vom Mathematiklernen. Während das Modell der Subjektiven Erfahrungsbereiche und die AOT Perspektive ausschließlich auf einer deskriptiven Ebene arbeiten, um das Handeln der Lernenden zu erklären, steht im Grundvorstellungskonzept der mathematische Inhalt als Kern didaktischen Handelns im Vordergrund. Ziel ist es, vom mathematischen Inhalt ausgehend Grundvorstellungen als normative Kategorien zu entwickeln, die der Erfahrungswelt der Lernenden entsprechen und gleichzeitig den mathematischen Kern sachadäquat repräsentieren. Grundvorstellungen sind somit prototypische mentale Modelle, die es Lernenden ermöglichen sollen mathematische Begriffe, Verfahren und Strukturen zu verstehen und diese in Sachzusammenhängen anzuwenden. Der stoffdidaktischen Analyse des mathematischen Kerns wird eine deskriptive Ebene der individuellen Vorstellungen der Lernenden gegenübergestellt. Durch die Analyse von Bearbeitungsprozessen der Lernenden sollen mit inhaltsanalytischen und interpretativen Methoden die tatsächlichen Vorstellungen und Erklärungsmodelle der Lernenden rekonstruiert werden, um Divergenzen zwischen den normativ angezielten Grundvorstellungen zu identifizieren und Maßnahmen zur konstruktiven Unterstützung von Lernprozessen und auch dem Transfer auf neue Anwendungszusammenhänge abzuleiten.

In diesem Zusammenhang spannt das Grundvorstellungskonzept einen Rahmen auf zur Analyse der Handlungen von Lernenden in Hinsicht auf das didaktische Ziel 
der Ausbildung von sachadäquaten Grundvorstellungen als prototypische mentale Repräsentationen mathematischer Inhalte, die es ermöglichen sollen entsprechende Strukturen in Sachzusammenhängen zu erfassen und mathematische Begriffe, Verfahren und Beziehungen anzuwenden.

Die didaktischen Analysen zur Entwicklung des Bruchzahlbegriffs machen deutlich, dass die Ausbildung eines tragfähigen und flexiblen Verständnisses von Bruchzahlen verschiedene Transferprozesse erfordert. Diese Transferprozesse können auf normativer Ebene, d.h. auf Grundlage sachanalytischer und stoffdidaktischer Überlegungen in der Übertragung von spezifischen Begriffsaspekten, Verfahren und Struktureigenschaften auf Aufgabenebene beschrieben werden und charakterisieren wichtige Schritte im Rahmen einer langfristigen und sukzessiven Entwicklung von Grundvorstellungen zu Bruchzahlen. Der Prozess des Transfers von Begriffsaspekten, Verfahren und Strukturen beruht dabei im Wesentlichen auf den individuellen Erklärungsmodellen der Lernenden, die durch Transferprozesse ausgeschärft, erweitert und miteinander vernetzt werden sollen.

Der umfassende Korpus an empirischen Untersuchungen beschreibt ausführlich verschiedene Hürden, Schwierigkeiten und Problembereiche in der Entwicklung des Bruchzahlbegriffs, die in vielen Fällen auf fehlerhafte Übertragungen von Struktureigenschaften der natürlichen Zahlen auf die Bruchzahlen (negativen Transfer), das Ausbleiben von Transfer sowie fehlerhafte Anpassungen von Struktur- und Operationskonzepten beschrieben werden, so dass notwendige Grundvorstellungserweiterungen und Grundvorstellungsanpassungen ausbleiben und Fehlvorstellungen und fehlerhafte Strategien entwickelt werden.

\section{Forschungsdesiderata}

Vor dem Hintergrund des Grundvorstellungskonzepts ist es möglich auf normativer Ebene Transferprozesse auf der Basis sachanalytischer Überlegungen in Hinsicht auf ein didaktisches Ziel zu beschreiben. Mit Blick auf die Entwicklung des Bruchzahlbegriffs als Zahlbereichserweiterung von den natürlichen Zahlen auf die positiven rationalen lassen sich folgende Forschungsdesiderata herausstellen:

Transferprozesse in der Ausbildung von Grundvorstellungen zu Bruchzahlen: Die Vielzahl der vorliegenden quantitativen Studien zum Bruchzahlbegriff beschreiben und vergleichen Kompetenzen und Leistungsstände der Lernenden zu einem bestimmten Zeitpunkt. In qualitativen Untersuchungen werden zudem individuelle Erklärungsmodelle und Vorstellungen der Lernenden analysiert und auf Grundlage der beobachteten Bearbeitungsstrategien und Argumentationsstrukturen vornehmlich der zum Zeitpunkt der Erhebung aktuelle Stand der Konzeptentwicklung festgestellt. Es finden sich nur wenige empirische Studien, die die 
längerfristige Entwicklung von Grundvorstellungen zu Bruchzahlen auf individueller Ebene untersuchen. Vor diesem Hintergrund finden sich bisher keine spezifischen Untersuchungen von Transferprozessen in der Entwicklung von Grundvorstellungen zu Bruchzahlen. Die Analyse von Transferprozessen verspricht eine hochauflösende Sicht auf die Begriffsentwicklung der Lernenden zu ermöglichen, auf deren Grundlage individuelle Entwicklungsverläufe im Aufbau tragfähiger und flexibler Vorstellungen beschrieben und Unterstützungsmaßnahmen abgeleitet werden können.

Entwicklung von Fehlvorstellungen und Fehlerstrategien durch ausbleibende und negative Transferprozesse: Der empirische Korpus zur Entwicklung des Bruchzahlbegriffs beschreibt eine Vielzahl von fehlerhaften Denkmustern bzw. Fehlvorstellungen und Fehlerstrategien, die Lernende entwickeln. In vielen Fällen werden die Probleme der Lernenden auf fehlerhafte Übertragungen von Struktureigenschaften der natürlichen Zahlen auf die Bruchzahlen erklärt und auf die unzureichende Ausbildung von Grundvorstellungen zurückgeführt. Die Entwicklung von Fehlvorstellungen und Fehlerstrategien wird dabei zumeist als Ergebnis ausbleibenden oder negativen Transfers im Anschluss an eine Unterrichtseinheit oder mit zeitlichem Abstand zu einer Unterrichtseinheit festgestellt. Somit ermöglichen nur wenige Studien einen Einblick in die tatsächlichen Entstehungsgeschichte. Es finden sich zum Beispiel nur wenige Erkenntnisse darüber, ob die Schwierigkeiten bereits zu Beginn der Einführung von Bruchzahlen entstehen oder ob sie durch Übergeneralisierungen über den Verlauf des Unterrichts entwickelt werden.

Open Access Dieses Kapitel wird unter der Creative Commons Namensnennung 4.0 International Lizenz (http://creativecommons.org/licenses/by/4.0/deed.de) veröffentlicht, welche die Nutzung, Vervielfältigung, Bearbeitung, Verbreitung und Wiedergabe in jeglichem Medium und Format erlaubt, sofern Sie den/die ursprünglichen Autor(en) und die Quelle ordnungsgemäß nennen, einen Link zur Creative Commons Lizenz beifügen und angeben, ob Änderungen vorgenommen wurden.

Die in diesem Kapitel enthaltenen Bilder und sonstiges Drittmaterial unterliegen ebenfalls der genannten Creative Commons Lizenz, sofern sich aus der Abbildungslegende nichts anderes ergibt. Sofern das betreffende Material nicht unter der genannten Creative Commons Lizenz steht und die betreffende Handlung nicht nach gesetzlichen Vorschriften erlaubt ist, ist für die oben aufgeführten Weiterverwendungen des Materials die Einwilligung des jeweiligen Rechteinhabers einzuholen.

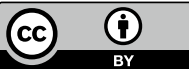

